\title{
A semilinear equation for the American option in a general jump market
}

\author{
KenNeTH H. KARLSEN AND Olli WALlin \\ Centre of Mathematics for Applications (CMA), University of Oslo, \\ P.O. Box 1053, Blindern, N-0316 Oslo, Norway \\ E-mail: kennethk@math.uio.no,olliw@math.uio.no
}

[Received 11 March 2008 and in revised form 26 September 2009]

\begin{abstract}
We study the pricing of American put and call options in a market with jumps. We extend and make rigorous previous work that characterizes the price as a solution of an integro-differential equation set on the whole domain. The equation closely resembles the equation for the corresponding European options, but involves an additional reaction term that depends on the American option value in a nonlinear, nonlocal and discontinuous manner. Thus standard theory for partial differential equations does not apply, and we give a proper definition of a viscosity solution of the equation. We then show that the characterization is well posed. In particular, we prove a strong comparison principle for the equation using an approach that overcomes some problems related to the appearance of integrals with respect to unbounded measures. In short, we extend the results in [20] to a general class of exponential additive models. The formulation constitutes a starting point for designing and analyzing "easy to implement" numerical algorithms for computing the value of an American option.
\end{abstract}

\section{Introduction}

We consider the pricing of American options under Lévy models [54, 56, 51, 53, 13, 12, 35, 63, 32, 50]. The traditional approaches to the solution of the American option problem can be divided into two main categories, namely those based on the free boundary problem formulation and those based on quasi-variational inequality formulation.

In the free boundary problem, one simultaneously looks for the value and a boundary that splits the domain into a continuation set, where the value satisfies a differential equation, and the stopping set, where the value is equal to a known function. The connection between the American option pricing problem and free boundary (or Stefan) problems was already given by Samuelson in the ground breaking article [65], with the mathematics worked out by McKean in the appendix [55]. The approach is tricky especially for pure jump processes, since the smooth fit principle typically assumed to hold at the free boundary often breaks down. In this case, the smooth fit should be replaced by a condition of continuous fit. Thus, when stating the problem one always needs to investigate whether continuous or smooth fit should be applied, which may be difficult. The break down of the smooth fit for Poisson processes was known already to McKean (see also Alili and Kyprianou [1] for a recent survey of these matters). We finally mention that Pham [61] showed that the free boundary formulation can be successfully applied for a strictly positive diffusion coefficient and a finite intensity jump process. He made the assumption that the riskless interest rate corrected by the jump risk should be nonnegative. Very recently, Bayraktar [15] proved that the value function is a classical solution without this condition (see also [16, 17, 72]).

The approach through solving quasi-variational inequalities was developed by Bensoussan and Lions [18], [19] and applied to American option pricing by Jaillet et al. [59]. In a quite general 
set up, Pham [62] used this approach to show that the American option price can be characterized as the unique viscosity solution of a fully nonlinear variational inequality (see also [2]). On the other hand, Lamberton and Mikous [52] proved that the value function is the unique distributional solution of the variational inequality. This result holds for all exponential Lévy processes. For a completely different approach that yields regularity estimates for the obstacle problem for the fractional Laplacian, see [25].

Even in the case of the classical Black and Scholes market, it seems hard to come to an exact and explicit formula for the American put price, such that numerical values could be computed efficiently. However, it is well known that the price can be expressed quite explicitly in terms of the free boundary. In a recent article [74] Zhu presented an explicit formula as an infinite series in which the terms involve multiple integrals and special functions. This has great value for theoretical and back-testing purposes, but whether the expression gives an efficient tool for computation of numerical values is yet to be tested. Moreover, to our knowledge this formulation or other analytical approximation techniques have not been extended to the general class of exponential additive models (see [14], [27], [31], [74] and the references therein for analytical approximations). One therefore still has to resort to numerical discretization techniques to solve the problem, and since Brennan and Schwartz [24] there has been a lot of work to develop better methods for this purpose. The above frameworks of free boundary and quasi-variational inequalities lend themselves to different numerical schemes, which have advantages and shortcomings specific to the formulation. For these we refer to [57].

Our goal here is to extend a different formulation of the valuation problem carried out in [20], which started from the works of Jamshidian [46] and Kholodnyi [49]. We shall focus on American put options for which the payoff at exercise is given by $g_{p}(x)=(K-x)^{+}$, where $K$ is the strike price. Modifications needed to handle the case of a call option $g_{c}(x)=(x-K)^{+}$are also mentioned.

Roughly speaking, in our formulation we seek a function $v=v(t, x)$ solving the following semilinear partial integro-differential equation (PIDE):

$$
\mathcal{L}_{B S} v(t, x)+B(t, x, v)=-q(t, x, v)
$$

where $x \geqslant 0, t \in[0, T), \mathcal{L}_{B S}$ is a differential operator, and $B$ is an integral operator.

The nonlinear reaction term $q$ takes the form

$$
q(t, x, v)= \begin{cases}0, & g(x)-v(t, x)<0 \\ c(t, x, v), & g(x)-v(t, x) \geqslant 0\end{cases}
$$

for a cash flow function $c=c(t, x, v)$ defined as

$$
c(t, x, v)=\left(r K-d x-D_{g}(t, x, v)\right)^{+},
$$

for the put option and

$$
c(t, x, v)=\left(d x-r K-D_{g}(t, x, v)\right)^{+}
$$

for the call option, where $D_{g}$ is another integral operator depending on the payoff $g$ and $r \geqslant 0$, $d \geqslant 0$ are the constant interest and dividend rates, respectively. In addition, the value satisfies the terminal condition $v(T, x)=g(x)$. The exact form of the operators can be found in Sections 5 and 6 We call this the semilinear Black and Scholes (SLBS) equation. In the rest of the article, we shall drop the dependence of the integral operator $D=D_{g}$ on the payoff $g$, as we will mainly deal with the case of a put option. 
One of our motivations for studying the SLBS equation is that it allows designing and analyzing "easy to implement" numerical schemes. Notice that we could regain the PIDE for the price of a European option by simply taking away the reaction term $q$. In fact, any solver for the European price can be turned into a solver for the American price using the semilinear formulation. Thus, the equation is also referred to as the nonhomogeneous Black and Scholes equation in the literature. Simple examples of such schemes for the Black and Scholes market were studied and analyzed in Benth et al. [21]. The SLBS equation is also related to so called penalty schemes, which have been studied in connection to American option pricing in [38, 75, 58], as some of these schemes can be seen as approximations to the semilinear equation [1.1]. We refer to [21] for a rigorous derivation of this connection in the pure diffusion case. The design and analysis of numerical schemes for the nonlocal semilinear equation (1.1), which is outside the scope of this paper, will be the topic of future work. Compared to the pure diffusion case [21], one added difficulty is related to the discretization of highly nonlocal and singular integral operators. However, this is currently an active area of research and there are several methods to choose from (cf. for example [32]).

Notice that our equation is set in the whole domain $[0, T) \times \mathbb{R}_{+}$, so we do not need to determine a free boundary. In addition, there are no side constraints as in the quasi-variational formulation. However, the nonlinearity $v \mapsto q(t, x, v)$ is discontinuous, which raises the question how one should interpret the semilinear equation. Guided by the dynamic programming principle, we suggest a suitable definition of a viscosity solution (see [34], [73]) for the semilinear PIDE (1.1). Even if the application of viscosity solutions theory for control problems is standard by now, dealing with a discontinuous operator is not. Here we apply ideas from [20], which again draws from the work of Ishii [41] for first order differential operators. However, the situation is now significantly more complicated than in the pure diffusion case [20]. The function $q$ is not only discontinuous but it also depends on an integral operator in a highly nonlinear way, which makes the overall analysis very delicate. In particular, this remark applies to the proof of the comparison principle (uniqueness), which is substantially more difficult than in the diffusion case.

One of the main contributions here is our proof of the comparison principle for viscosity solutions of the SLBS equation. The Lévy measure of the integral operators in the equation may have a second order singularity at zero, so it is not always obvious whether such integrals are well defined. This makes the application of the maximum principle for semicontinuous functions (also known as Ishii's lemma, see [33] and [34]) in connection with integro-differential equations problematic, and there has been increasing interest in this issue. We refer the interested reader to [45, 4, 5, 10] for details.

For completeness, let us also mention that the notion of viscosity solutions for integrodifferential equations without a diffusion operator, which is simpler, was first studied by Soner [70] and Sayah [67, 68], and then by many others.

To gain more insight, we go back to the original approach of using semiconvex approximations. This dates back to the early work of Jensen, Ishii and Lions in [47], [42] and [43] (see also the textbook of Yong and Zhou [73]). For an adaption to the nonlocal setting, our main source of inspiration is Jakobsen and Karlsen [45]. In [45] it is explained why the classical results [42, 43] do not apply directly to equations with general, second order singular integral operators.

We cannot directly apply the abstract "maximum principle" of [45] since part of our nonlinearity is discontinuous and at the same time has a nonlocal dependence that is not covered by the standard assumptions utilized in [45]. Consequently, we will have to work out a detailed proof properly adapted to the present context. In doing so we have chosen an approach that is somehow 
different from the one used in [45] and seems (modestly) original. First, we do not rely on an abstract maximum principle as the proof uses the second order conditions for maxima from standard multivariable calculus. Moreover, and more importantly, there is no need to decompose the integral operators into several parts separating the singular region from the rest, which to our knowledge is a new feature compared to all the previous work done on Hamilton-Jacobi equations involving singular integral terms (cf. for example [4, 5, 10, 45, 62, 67, 68]). Finally, we are allowing the Lévy measure to have some (mild form of) time dependence, where the motivation is to allow for a more general class of additive processes that is relevant for applications to finance.

A similar equation has been used in several articles to study numerical methods under various model assumptions. For example, essentially the same PIDE in the case of variance gamma has already appeared in Hirsa and Madan [40] (see also Carr and Hirsa [26] where a transformed equation is used in connection with model calibration). In addition, in a series of papers [28], [29], [30], Chiarella and Ziogas combine such an equation with the incomplete Fourier transform to derive new numerical schemes. In all of these articles, the equation is stated in the form of a free boundary problem. However, no rigorous theory is built. We argue that such a formulation cannot be stated on the whole domain because of lack of smoothness of the solution over the free boundary. Moreover, in our formulation no precomputation of a free boundary is needed in order to solve the option price.

Let us note that in a complete market setting (the pure diffusion case), the reaction term $q$ is nothing but the consumption density process of the writer of the option. Thus the equation should be interpreted as the infinitesimal version of the early exercise premium representation of the American option price. See the last section in [20] for a heuristic discussion of this point. Finally, while we do not study the perpetual case $T=+\infty$ here, one can see that the price of a perpetual option should satisfy an elliptic version of the semilinear equation.

The remainder of this article is organized as follows. In Section 2 we establish some notations, and Section 3 offers a brief introduction to exponential additive models. In Section 4 we review results on optimal stopping, and we show on a heuristic level how to derive a semilinear equation for the American put option price in Section 5. Then we set up a rigorous definition of a solution to this equation in Section 6 via viscosity solution theory. Finally, Sections 7 and 8 give the main results on well-posedness of the American option value in our framework.

\section{Notation}

For a set $A \subset \mathbb{R}^{N}, N \in \mathbb{N}$, let $B(A)$ be any class of real-valued functions on $A$. We will denote by $B_{1}(A)$ the subclass of functions with at most linear growth, that is, functions $f \in B(A)$ such that

$$
f(x) \leqslant L(1+|x|)
$$

for some $L>0$. We recall that for every locally bounded function $f: A \rightarrow \mathbb{R}$, its upper and lower semicontinuous envelopes, denoted by $f^{*}$ and $f_{*}$ respectively, are defined as

$$
h^{*}(x):=\limsup _{y \rightarrow x} h(y), \quad h_{*}(x):=\liminf _{y \rightarrow x} h(y) .
$$

A locally bounded function $f: A \rightarrow \mathbb{R}$ is said to be upper semicontinuous if $f^{*} \leqslant f$ and lower semicontinuous if $f_{*} \geqslant f$. In particular,

$$
H^{*}(x):=H(x)=\left\{\begin{array}{ll}
0 & \text { if } x<0, \\
1 & \text { if } x \geqslant 0,
\end{array} \quad H_{*}(x):= \begin{cases}0 & \text { if } x \leqslant 0 \\
1 & \text { if } x>0\end{cases}\right.
$$


are the upper and lower semicontinuous envelopes, respectively, of the Heaviside function $H$. If $h$ is both upper and lower semicontinuous then it is continuous. We denote the sets of upper and lower semicontinuous functions by $U S C(A)$ and $L S C(A)$, respectively. As usual, we denote by $C(A)$ the class of continuous functions on $A$. In addition we denote by $U S C_{1}^{+}(A)\left[L S C_{1}^{+}(A)\right]$ the class of non-negative functions belonging to $\operatorname{USC}(A)[\operatorname{LSC}(A)]$ and satisfying (2.1). In what follows, we adopt the notations $\mathbb{R}_{+}:=[0, \infty), \mathcal{O}_{T}=[0, T) \times[0, \infty)$, and $\overline{\mathcal{O}_{T}}=[0, T] \times[0, \infty)$. We say that a function $v$ is $C^{1,2}$ at the point $(t, x) \in \mathcal{O}_{T}$ if $v$ is once continuously differentiable in $t$ and twice continuously differentiable in $x$. Moreover, we say $v$ is $C_{1}^{1,2}$ at $(t, x)$ if, in addition, it has at most linear growth so that 2.1 is satisfied. Finally, $C_{1}^{1,2}\left(\mathcal{O}_{T}\right)$ is the class of functions that are $C_{1}^{1,2}$ at all points $(t, x) \in \mathcal{O}_{T}$.

\section{Exponential additive processes}

In this section we briefly review the class of exponential additive processes we will use to model stock price evolution. We rely largely on [50], which also introduces some financial applications. General references for the special case of Lévy processes are [3], [22], [66], and financial applications are discussed, for example, in [32], [36] and [63]. Properties of additive processes can be found in Chapter 2 of [66] and Chapter 14 of [32]. Relations to semimartingales are detailed in [44]. Let us first, however, note that financial models driven by such processes are in general incomplete, meaning that not all derivatives can be perfectly replicated by dynamic trading in the underlying. This then implies that there are in fact an infinite number of equivalent martingale measures to choose from, each giving an arbitrage free pricing rule. While there are several theoretical and practical ways to choose one, we simply assume in this paper that a pricing measure $\mathbb{Q}$ is given and all the dynamics considered henceforth are under this measure.

Let $(\Omega, \mathcal{F}, \mathbb{F}, \mathbb{Q})$ be a filtered probability space satisfying the usual conditions. A stochastic process $X=(X(t))_{t \in[0, T]}$ on $\mathbb{R}$ is called additive if it is stochastically continuous with RCLL (i.e., right continuous with left limits) sample paths and independent increments. Given such a process $X$ we assume that $\mathbb{F}=\mathbb{F}^{X}$, i.e., we take the filtration to be the completed natural filtration generated by $X$.

Some additive processes are not semimartingales: any deterministic, continuous function with infinite variation provides a trivial example of this. This is not desirable since we lose Itô's formula and further, we might introduce models with arbitrage opportunities. It is furthermore clear that excluding such peculiarities from our modeling framework is not restricting us in building realistic models. This motivates us to work with a slightly more restricted class of processes.

DEFINITION 1 The process $X$ has independent increments with absolutely continuous characteristics if for every $t \in[0, T)$ the distribution of $X(s)-X(t), t<s$, is independent of $\mathcal{F}_{t}$ and the characteristic function $\Phi_{t}(u):=\mathbb{E}\left[\exp \left(i u X_{t}\right)\right]$ of $X(t)$ is given by

$$
\Phi_{t}(u)=\exp \left\{\int_{0}^{t}\left(i u b(s)-\frac{1}{2} u^{2} \sigma^{2}(s)+\int_{\mathbb{R}}\left(e^{i u z}-1-i u z\right) v_{s}(\mathrm{~d} z)\right) \mathrm{d} s\right\} .
$$

Here $b, \sigma$ are measurable functions on $[0, T]$ and for each $s, v_{s}(\cdot)$ is a Borel measure on $\mathbb{R}$ such that $v_{s}(0)=0$,

$$
\int_{0}^{T}\left(|b(s)|+\left|\sigma^{2}(s)\right|+\int_{\mathbb{R}}\left(z^{2} 1_{|z| \leqslant 1}(z)+\exp (2 z) 1_{\{|z|>1\}}(z)\right) v_{s}(\mathrm{~d} z)\right) \mathrm{d} s<\infty .
$$


A stochastic process with independent increments and absolutely continuous characteristics is henceforth abbreviated as PIIAC.

Stochastic continuity of $X$ is actually implied by equation 3.1). Furthermore, a PIIAC is an additive process in law and has an RCLL modification which is also a semimartingale (see [50, p. 8]). We will always work with this RCLL version of $X$. Finally, it follows from Corollary 4.18 in [44, p. 107] that $X$ is also quasi-left-continuous, i.e., left continuous over stopping times.

In the definition above, the integrability condition on the tails of the measure $v_{s}(\cdot)$ is stronger than what is usually given. This assumption is related to our proof of the comparison principle for solutions of (1.1), and also implies that the price process is square-integrable. Notice, however, that we allow for fully general behavior of the measure near zero, and a possibly vanishing $\sigma$ to include pure jump processes with infinite activity. In addition, to make it easier to take limits we will require that $b$ and $\sigma$ are continuous functions on $[0, T]$, and $v_{s}(\cdot)=\rho(s) v(\cdot)$ for a continuous, nonnegative function $\rho$ and a time independent measure $v(\cdot)$. Then we also see that if $f=f(z)$ is a continuous function and $\kappa \geqslant 0$ is such that

$$
\int_{|z|>\kappa} f(z) v_{s}(\mathrm{~d} z)<\infty \quad \text { for all } s \in[0, T],
$$

then

$$
\lim _{s \rightarrow t} \int_{|z|>\kappa} f(z) v_{s}(\mathrm{~d} z)=\int_{|z|>\kappa} f(z) v_{t}(\mathrm{~d} z) .
$$

Let $J_{X}(\mathrm{~d} s, \mathrm{~d} z)$ denote the (random) jump measure associated to the RCLL process $X$ (see [44]) and let

$$
\tilde{J}_{X}(\mathrm{~d} s, \mathrm{~d} z)=J_{X}(\mathrm{~d} s, \mathrm{~d} z)-v_{s}(\mathrm{~d} z) \mathrm{d} s
$$

denote the compensated jump measure. Given our assumptions on $X$, it is a special semimartingale and thus has canonical representation

$$
X(t)=\int_{0}^{t} b(s) \mathrm{d} s+\int_{0}^{t} \sigma(s) \mathrm{d} W(s)+\int_{0}^{t} \int_{\mathbb{R} \backslash\{0\}} z \tilde{J}_{X}(\mathrm{~d} s, \mathrm{~d} z),
$$

where $W$ is a Brownian motion (see [44, II.2.34] or [50]). Then we model the dynamics of the stock price $(S(t))_{t \in[0, T]}$ under the martingale measure $\mathbb{Q}$ as

$$
S(t)=S(0) \exp ((r-d) t+X(t)) .
$$

Assuming that $\mathbb{Q}$ is a martingale measure means that the discounted price process with dividends

$$
\bar{S}(t)=e^{-(r-d) t} S(t)=S_{0} \exp (X(t))
$$

has to be a (local) martingale under $\mathbb{Q}$. Then the price model is free of arbitrage. A necessary and sufficient condition for the martingale property to hold is that, for each $t$, the characteristics $\left(b(t), \sigma^{2}(t), v_{t}(\cdot)\right)$ satisfy

$$
b(t)+\frac{\sigma^{2}(t)}{2}+\int_{0}^{t} \int_{\mathbb{R}}\left(e^{z}-1-z\right) v_{s}(\mathrm{~d} z) \mathrm{d} s=0 .
$$

For example, in the Black-Scholes model $d=0, v \equiv 0, \sigma(t) \equiv \sigma$ so we must have $b(t) \equiv-\frac{1}{2} \sigma^{2}$, which combined with 3.2 gives the risk neutral drift $r-\frac{1}{2} \sigma^{2}$ for the log-prices of this fundamental 
model. Finally, we will assume without loss of generality that the the model satisfies the natural condition

$$
\mathbb{Q}^{t, x}\left(\tau_{A} \leqslant T\right)>0
$$

for any $t<T, x>0$, and any open set $A$ such that $\operatorname{cl}(A) \subset(0,+\infty)$ where $\tau_{A}:=\inf \{u \in[t, T]$ : $S(u) \notin A\}$ is the first exit time from the set. This ensures that the option value is always strictly positive before expiration. Detailed study of when (3.4) holds for Lévy processes can be found in [66, Theorem 24.10, p. 152]. We only mention that it is sufficient that either $\sigma \neq 0$, or that the the process has both positive and negative jumps, so basically all popular models have this property. While we thus exclude spectrally one-sided processes, we mention that we could handle these as well by simply truncating the space domain. In our setting we also have time-dependent coefficients: we need to assume that either $\sigma(\cdot)$ or $\rho(\cdot)$ is bounded away from zero.

In the next section, we will use that by independence of increments of $X$ the price process $S$ is a strong Markov process. This is usually proved for Lévy processes only, but it holds for the class PIIAC also as is argued on p. 267 in [39]. Since $X$ is a real-valued, quasi-left-continuous strong Markov process with RCLL paths on $[0, T]$, it is a standard Markov process in the sense of Blumenthal and Getoor [23].

\section{Optimal stopping of Markov processes}

Let $S$ be a standard Markov process with transition function $(s, y) \mapsto \mathbb{Q}^{s, y}$ (see [64] or [69] for rigorous definitions). Then, for each fixed $(t, x), \mathbb{Q}^{t, x}$ is a probability measure such that $\mathbb{Q}^{t, x}(S(t)=$ $x)=1$, and we denote by $\mathbb{E}^{t, x}$ the expectation under this measure. Given $g \in C_{1}\left(\mathbb{R}_{+}\right), g \geqslant 0$, we wish to find

$$
v(t, x)=\sup _{t \leqslant \tau \leqslant T} \mathbb{E}^{t, x}\left[e^{-r(\tau-t)} g(S(\tau))\right],
$$

where, in particular, $g(x)=(K-x)^{+}$for the put option and $g(x)=(x-K)^{+}$for the call option. In the financial context, any stopping time is an exercise strategy of the American option. It is then of natural interest also to look for a stopping time $\tau_{0}$ which achieves the supremum. If such a $\tau_{0}$ exists, it is called an optimal stopping time.

From the definition it follows immediately that $v \geqslant g, v(T, x)=g(x)$, and the optional sampling theorem together with the martingale property of $\bar{S}$ implies that

$$
v(t, x) \leqslant L(1+x)
$$

for general $g \in C_{1}\left(\mathbb{R}_{+}\right)$, or $v(t, x) \leqslant K$ for the put option and $v(t, x) \leqslant x$ for the call option. Also, if $g \geqslant 0$ is not identically zero, then it follows from (3.4) that $v>0$ on $(0,+\infty)$. To apply general theorems in optimal stopping, it is required that the process $g\left(S_{t}\right)$ satisfies some stronger integrability conditions. We follow the standard assumption from [69, p. 115] that

$$
\mathbb{E}\left[\sup _{t \in[0, T]}\left|g\left(S_{t}\right)\right|\right]<\infty .
$$

The next proposition is used heavily both in the next section when deriving the semilinear equation (1.1) and in Section 7 where it is used to show that the value function $v$ is a viscosity solution of (1.1). For $\epsilon \geqslant 0$, define the stopping time

$$
\tau_{\epsilon}:=\inf \{u \in[t, T] \mid v(u, S(u)) \leqslant g(S(u))+\epsilon\} .
$$


PROPOSITION 1 (Dynamic programming principle (DPP) [69, 60])

(i) For all stopping times $\theta$ taking values in $[t, T]$, we have

$$
v(t, x) \geqslant \mathbb{E}^{t, x}\left[e^{-r(\theta-t)} v(\theta, S(\theta))\right] .
$$

(ii) Any stopping time $t \leqslant \theta \leqslant \tau_{\epsilon}$ satisfies

$$
v(t, x)=\mathbb{E}^{t, x}\left[e^{-r(\theta-t)} v(\theta, S(\theta))\right] .
$$

(iii) $\tau_{0}$ is an optimal stopping time for $g(S(t))$, and $e^{-r\left(u \wedge \tau_{0}-t\right)} v\left(u \wedge \tau_{0}, S\left(u \wedge \tau_{0}\right)\right)$ is a martingale.

In addition to growth properties, we need the solutions to be continuous to apply viscosity solution theory. The following result can be proved as in Pham [62, p. 10, Prop. 3.3].

PROPOSITION 2 Suppose the Markov process is an exponential additive process as given in Section 3. Suppose furthermore the pay-off function $g \in C_{1}\left(\mathbb{R}_{+}\right), g \geqslant 0$, is Lipschitz. Then the value function $v$ in 4.1 is continuous.

\section{Formal derivation of the semilinear equation}

Next we will proceed to derive the semilinear Black-Scholes equation for the American put option $g(x)=(K-x)^{+}$. Our derivation here is only formal, rigorous definitions and proofs follow in the subsequent sections. We assume in particular that $v \in C^{1,2}\left(\mathcal{O}_{T}\right)$.

Let $S$ be an exponential PIIAC process under the risk neutral measure as defined in Section 3 , and let $(t, x) \in \mathcal{O}_{T}$. Applying Itô's formula to $Y(s):=e^{-r(u-t)} v\left(u, S^{t, x}(u)\right), u \in[t, T]$, yields

$$
\begin{aligned}
\mathrm{d} Y(s)= & e^{-r(u-t)}\left[\mathcal{L}_{B S} v\left(u, S^{t, x}(u)\right)+B\left(u, S^{t, x}(u), v\right)\right] \mathrm{d} u \\
& +e^{-r(u-t)} \sigma(u) S(u) \partial_{x} v\left(u, S^{t, x}(u)\right) \mathrm{d} W_{u} \\
& +e^{-r(u-t)} \int_{\mathbb{R}}\left[v\left(u, S(u-) e^{z}\right)-v(u, S(u-))\right] \tilde{J}_{X}(\mathrm{~d} t, \mathrm{~d} z),
\end{aligned}
$$

where $\mathcal{L}_{B S} v(u, s)=\partial_{u} v(u, s)+(r-d) s \partial_{s} v(u, s)+\frac{1}{2} \sigma^{2}(t) s^{2} \partial_{s}^{2} v(u, s)-r v(u, s)$ and

$$
B(u, s, v)=\int_{\mathbb{R}}\left[v\left(u, s e^{z}\right)-v(u, s)-s\left(e^{z}-1\right) \partial_{s} v(u, s)\right] v_{u}(\mathrm{~d} z) .
$$

This integral is well defined for $v \in C^{1,2}\left(\mathcal{O}_{T}\right)$, as can be seen by Taylor's theorem and the fact that the measure $v_{t}(\cdot)$ integrates $\left(e^{z}-1\right)^{2}$ on $\mathbb{R} \backslash\{0\}$. The stochastic integrals are true martingales with zero expectation, at least up to an exit time from a small neighborhood of $(t, x)$. Taking expectations on both sides and using inequality 4.4 then gives

$$
\mathcal{L}_{B S} v(t, x)+B(t, x, v) \leqslant 0
$$

everywhere for the value function. Furthermore, equation 4.5 implies

$$
\mathcal{L}_{B S} v(t, x)+B(t, x, v)=0
$$

in the continuation region $\{v(t, x)>g(x)\}$. In the exercise region, $\mathcal{L}_{B S} v(t, x)+B(t, x, v)$ is nonpositive. However, as we will see, it is possible to derive a lower bound for $\mathcal{L}_{B S} v(t, x)+$ 
$B(t, x, v)$ in this region as well. To this end, fix a point $(t, x)$ in the exercise region. Since $v(t, x)=g(x)$ and $v \geqslant g$ everywhere, $(t, x)$ is a global maximizer of $g-v$. In what follows, we consider the put option. Because $v>0$ and $g(x)=0$ for $x \geqslant K$, we conclude that $x<K$, where $g$ is smooth. We must have $\partial_{t} v(t, x)=0, \partial_{x} v(t, x)=-1, \partial_{x}^{2} v(t, x) \geqslant 0$. Recalling the definition of $H_{*}$, the integral term has the value

$$
\begin{aligned}
B(t, x, v) & =\int_{\mathbb{R}}\left(v\left(t, x e^{z}\right)-\left(K-x e^{z}\right)\right) v_{t}(\mathrm{~d} z) \\
& =\int_{\mathbb{R}} H_{*}\left(v\left(t, x e^{z}\right)-\left(K-x e^{z}\right)\right)\left(v\left(t, x e^{z}\right)-\left(K-x e^{z}\right)\right) v_{t}(\mathrm{~d} z),
\end{aligned}
$$

where the last equality follows from noticing that $v(t, x e z) \geqslant g\left(x e^{z}\right) \geqslant K-x e^{z}$. Thus we have discovered that

$$
\begin{aligned}
\mathcal{L}_{B S} v(t, x) & +B(t, x, v) \\
\geqslant & -\left(r K-d x-\int_{\mathbb{R}} H_{*}\left(v\left(t, x e^{z}\right)-\left(K-x e^{z}\right)\right)\left(v\left(t, x e^{z}\right)-\left(K-x e^{z}\right)\right) v_{t}(\mathrm{~d} z)\right) .
\end{aligned}
$$

However, since 5.1 tells us that the right hand side in the above inequality is nonpositive, we conclude that

$$
\begin{aligned}
& \mathcal{L}_{B S} v(t, x)+B(t, x, v) \\
& \quad \geqslant-\left(r K-d x-\int_{\mathbb{R}} H_{*}\left(v\left(t, x e^{z}\right)-\left(K-x e^{z}\right)\right)\left(v\left(t, x e^{z}\right)-\left(K-x e^{z}\right)\right) v_{t}(\mathrm{~d} z)\right)^{+}
\end{aligned}
$$

when $v(t, x)=g(x)$.

Let us now collect the information revealed by the derivations and the remark above into a single equation, without explicitly using the concept of a free boundary. For $v$, let

$$
D(t, x, v):=\int_{\mathbb{R}} H_{*}\left(v\left(t, x e^{z}\right)-\left(K-x e^{z}\right)\right)\left(v\left(t, x e^{z}\right)-\left(K-x e^{z}\right)\right) v_{t}(\mathrm{~d} z) .
$$

It is not obvious when $D$ has a finite value for a given function $v$ and point $(t, x)$. We treat this question in detail in the next section. Now, we define the cash flow function

$$
c(t, x, v)=(r K-d x-D(t, x, v))^{+}
$$

and the reaction term

$$
q(t, x, v)=H(g(x)-v(t, x)) c(t, x, v) .
$$

Then the semilinear Black and Scholes partial integro-differential equation for the value function of an American option is

$$
\mathcal{L}_{B S} v(t, x)+B(t, x, v)=-q(t, x, v) .
$$

As noted in Section 4 , the value function also satisfies the terminal condition

$$
v(T, x)=g(x)
$$


We should explain in what sense exactly (5.7) can be taken as an equality. On the one hand, if $(t, x)$ belongs to the interior of the stopping region $\mathcal{S}$, then $v(s, y)=K-y$ in a neighborhood of $(t, x)$ and the inequality in $(5.3)$ becomes an equality. On the other hand, the continuation region is known to be open, non-empty and connected. Thus (5.7) holds almost everywhere on $\mathcal{O}_{T}$. However, this characterization is not unique without further knowledge of the behavior of $v$ at the boundary of $\mathcal{C}$. In the viscosity solutions approach presented in the next section the inequalities derived above are built into the definition of a solution and no such information is needed.

One can derive the equation for the call option in the same way. We point out that for the call option, it is well known that if there are no dividends $(d=0)$ we have

$$
v(t, x)=\mathbb{E}^{t, x}\left[e^{-r(T-t)} g(S(T))\right]
$$

That is, the value of the American call option under $\mathbb{Q}$ equals the value of the European option under $\mathbb{Q}$, and it is not optimal to exercise before the terminal time $T$.

\section{Viscosity solutions}

In the previous section, we derived a partial integro-differential equation for the value of an American put. However, it is known that the value function is, in general, not smooth. Also, the discontinuity of the nonlinear operator in the solution $v$ is nonstandard, and we need to interpret equation (5.7) in a proper way. To deal with these problems, we follow [20, 41] and use the framework of viscosity solutions theory. In addition, care has to be taken to ensure that the integral term $D$ appearing in the cash flow function $c$ is well defined.

We discuss the finiteness of the integral $D$ after giving our definition of viscosity solutions. We give two definitions of the source term $q$, namely

$$
q^{*}(t, x, v)=H^{*}(g(x)-v(t, x))(r K-d x-D(t, x, v))^{+}
$$

and

$$
q_{*}(t, x, v)=H_{*}(g(x)-v(t, x))(r K-d x-D(t, x, v))^{+} .
$$

We warn the reader that these are definitions, and despite the notation we do not yet claim any semicontinuity properties of $q^{*}, q_{*}$ but instead return to these questions later in this section. Let us note that these definitions make sense, even if the value of the integral $D$ is not finite. By definition, the integrand of $D$ is nonnegative everywhere, and thus the integral is well defined in the Lebesgue sense even though it could take infinite values. We can extend the domain of definition for $(\cdot)^{+}$to the extended real line $[-\infty,+\infty]$ by setting $(-\infty)^{+}=0$. It will be shown shortly that $D$ is finite in the region where $g(x) \geqslant v(t, x)$. In the region where $g(x)<v(t, x)$ (and $D$ could be infinite so that $c$ vanishes), $H^{*}$ and $H_{*}$ vanish.

DEFINITION 2 (i) A nonnegative function $v \in U S C_{1}^{+}\left(\overline{\mathcal{O}_{T}}\right)$ is a viscosity subsolution of 5.7) if and only if for all $\phi \in C_{1}^{1,2}\left(\overline{\mathcal{O}_{T}}\right)$ such that $v \leqslant \phi$ we have

$$
\mathcal{L}_{B S} \phi(t, x)+B(t, x, \phi)+q^{*}(t, x, \phi) \geqslant 0
$$

whenever $\phi(t, x)=v(t, x)$ and $v(t, x)>0$. If, in addition, $\left.v\right|_{\{t=T\}} \leqslant g$ on $[0, \infty)$, then $v$ is a viscosity subsolution of the terminal problem 5.7)-5.8. 
(ii) A nonnegative function $v \in L S C_{1}^{+}\left(\overline{\mathcal{O}_{T}}\right)$ is a viscosity supersolution of 5.77 if and only if for all $\phi \in C_{1}^{1,2}\left(\overline{\mathcal{O}_{T}}\right)$ such that $v \geqslant \phi$ we have

$$
\mathcal{L}_{B S} \phi(t, x)+B(t, x, \phi)+q_{*}(t, x, \phi) \leqslant 0
$$

whenever $v(t, x)=\phi(t, x)$. If, in addition, $\left.v\right|_{\{t=T\}} \geqslant g$ on $[0, \infty)$, then $v$ is a viscosity supersolution of the terminal problem (5.7)- (5.8).

(iii) A nonnegative function $v \in C_{1}\left(\mathcal{O}_{T}\right)$ is a viscosity solution of 5.7 if and only if it is simultaneously a sub- and supersolution of (5.7). If, in addition, $\left.v\right|_{\{t=T\}}=g$ on $[0, \infty)$, then $v$ is a viscosity solution of the terminal problem $[5.77-5.8$.

Let us now discuss finiteness of the integral term $D$ (cf. (5.4)) in a slightly more general context than the above definition. Here we only require the test function to be continuous on $\overline{\mathcal{O}_{T}}$ and have at most linear growth. We have three cases to consider:

(i) If $v(t, x)=\phi(t, x)>g(x)$, then the integral $D$ is zero for both subsolutions and supersolutions.

(ii) If $v(t, x)=\phi(t, x)<g(x)$, then this together with nonnegativity of $v$ implies that $K-x=$ $g(x)>0$. By continuity $\phi\left(t, x e^{z}\right)-\left(K-x e^{z}\right)<0$ in a neighborhood of $z=0$ so the integrand vanishes near the possible singularity of $v_{t}(\cdot)$ for both sub- and supersolutions.

(iii) If $v(t, x)=\phi(t, x)=g(x)$, then the integral vanishes for supersolutions. For subsolutions, we only need to consider the case $x<K$ by the strict positivity assumption in the definition. Then

$$
\phi\left(t, x e^{z}\right)-\left(K-x e^{z}\right)=\phi\left(t, x e^{z}\right)-g\left(x e^{z}\right)
$$

in a neighborhood of $z=0$, so the integrand again vanishes.

REMARKS 3 (i) For the integrand of the operator $D$ we have

$$
\begin{aligned}
& H_{*}\left(v\left(t, x e^{z}\right)-\left(K-x e^{z}\right)\right)\left(v\left(t, x e^{z}\right)-\left(K-x e^{z}\right)\right) \\
& \quad=H\left(v\left(t, x e^{z}\right)-\left(K-x e^{z}\right)\right)\left(v\left(t, x e^{z}\right)-\left(K-x e^{z}\right)\right)=\left(v\left(t, x e^{z}\right)-\left(K-x e^{z}\right)\right)^{+} .
\end{aligned}
$$

In addition, if $v$ is strictly positive everywhere in $[0, T) \times(0,+\infty)$ then

$$
H_{*}\left(v\left(t, x e^{z}\right)-\left(K-x e^{z}\right)\right)=H_{*}\left(v\left(t, x e^{z}\right)-g\left(x e^{z}\right)\right) .
$$

In principle any of the above expressions could be used in the definition of $D$. However, since (numerical) approximations may take the value zero at least in some region, we want to allow for this possibility in our definition.

(ii) In recent papers, some other ways for writing the semilinear Black and Scholes equation have appeared, and we should point out the connection of our formulation to these. For this, we define the free boundary for the American put option as

$$
x_{p}(t):=\sup \{x: v(t, x)=g(x)\} .
$$

From equation 6.3, the integrand of $D$ is nonzero if and only if $z>\log \left(x_{p}(t) / x\right)$. Assuming we have verified the intuition that $v(t, x)=g(x)$ if and only if $x<x_{p}(t)$, the semilinear equation can 
be written in terms of the free boundary as

$$
\begin{aligned}
& \mathcal{L}_{B S} v(t, x)+B(t, x, v) \\
& \quad+1_{x<x_{p}(t)}(x)\left(r K-d x-\int_{\log \left(x_{p}(t) / x\right)}^{\infty}\left[v\left(t, x e^{z}\right)-\left(K-x e^{z}\right)\right] v_{t}(\mathrm{~d} z)\right)^{+}=0 .
\end{aligned}
$$

This equation (and its log-transformed version) are used, for example, in [40] and [26]. However, even in the case of the classical Black and Scholes market it is known that the second order derivative with respect to $x$ does not exist at the free boundary, so the equation cannot be interpreted in the classical sense on the whole domain. This has not been clearly pointed out in the previous literature, which mainly deals with numerical methods.

At this point, we note the following continuity properties for the integral terms. Let $v$ be a function and $\left\{\left(t_{k}, x_{k}\right)\right\}_{k \geqslant 1},(t, x)=\left(t_{0}, x_{0}\right)$, be points in $\mathcal{O}_{T}$ such that $v$ is $C_{1}^{1,2}$ at $(t, k)$ for $k \geqslant 0$ and $\left(t_{k}, x_{k}\right) \rightarrow(t, x)$ in $\mathcal{O}_{T}$. Suppose, in addition, that $v \in U S C\left(\mathcal{O}_{T}\right)$. By the sublinear growth and a general version of Fatou's Lemma (see [6, pp. 48 and 295])

$$
\limsup _{k \rightarrow \infty} B\left(t_{k}, x_{k}, v\right) \leqslant B(t, x, v) .
$$

Similarly, if $v \in \operatorname{LSC}\left(\mathcal{O}_{T}\right)$ we obtain

$$
\liminf _{k \rightarrow \infty} B\left(t_{k}, x_{k}, v\right) \geqslant B(t, x, v),
$$

and for $v \in C\left(\mathcal{O}_{T}\right)$,

$$
\lim _{k \rightarrow \infty} B\left(t_{k}, x_{k}, v\right)=B(t, x, v) .
$$

One can similarly verify that $(t, x) \mapsto D(t, x, v)$ is continuous in the relative topology of the set $A:=\{g-v \geqslant 0\} \cap\{v>0\}$, where we denote $\{f \geqslant 0\}=\{(t, x) \mid f(t, x) \geqslant 0\}$ for a function $f$. That is, if $\left(t_{k}, x_{k}\right) \rightarrow(t, x)$ in $A$, then $D\left(t_{k}, x_{k}, v\right) \rightarrow D(t, x, v)$. In the complement of $A$, the source term $q^{*}$ vanishes as $H^{*}(g(x)-v(t, x))$ does. Consequently, $q^{*}$ is upper semicontinuous. Similarly, we can prove that $q_{*}$ is lower semicontinuous. We collect these results in a lemma.

Lemma 4 Suppose $v \in U S C\left(\mathcal{O}_{T}\right)$. Then the mapping $(t, x) \mapsto q^{*}(t, x, v(t, x))$ is upper semicontinuous. Suppose $v \in L S C\left(\mathcal{O}_{T}\right)$. Then the mapping $(t, x) \mapsto q_{*}(t, x, v(t, x))$ is lower semicontinuous.

The next proposition lists some useful properties of the equation and its viscosity solutions. Especially, the monotonicity property of the nonlocal operators stated in (i) is crucial in many of the proofs that follow. Note that while the integral operator $B$ is clearly increasing in the nonlocal part $v$, the reaction term $q$ is decreasing in $v$, so the result in Proposition 5(i) is nontrivial.

Suppose $v \in C_{1}\left(\mathcal{O}_{T}\right)$ is $C^{1,2}$ at $(t, x)$. Then we say that $v$ satisfies the subsolution (supersolution) inequality in the classical sense at $(t, x)$ if we can replace the test function by $v$ everywhere in the corresponding inequalities (6.1) and 6.2).

PROPOSITION 5 (i) Suppose that $v_{1}, v_{2}$ are continuous functions with at most linear growth which are $C^{1,2}$ at $(t, x)$. If $v_{1}-v_{2}$ has a global minimum equal to zero at $(t, x)$, then

$$
B\left(t, x, v_{1}\right)+q\left(t, x, v_{1}\right) \geqslant B\left(t, x, v_{2}\right)+q\left(t, x, v_{2}\right),
$$


where $q$ stands for both $q_{*}$ (on the set $\left\{v_{2}>0\right\}$ ) and $q^{*}$. In addition,

$$
B\left(t, x, v_{1}+C\right)+q\left(t, x, v_{1}+C\right)=B\left(t, x, v_{1}\right)+q\left(t, x, v_{1}\right)
$$

for any constant $C \geqslant 0$.

(ii) Let $\left(t_{k}, x_{k}\right), k=1,2, \ldots$, and $(t, x)$ be such that $\left(t_{k}, x_{k}\right) \rightarrow(t, x)$ as $k \rightarrow \infty$. Suppose that there exists an associated collection of functions $v^{t_{k}, x_{k}}, v^{t, x}$ that are $C^{1,2}$ at $\left(t_{k}, x_{k}\right),(\mathrm{t}, \mathrm{x})$, respectively, and

$$
\partial_{x}^{n} v^{t_{k}, x_{k}}\left(t_{k}, x_{k}\right) \rightarrow \partial_{x}^{n} v^{t, x}(t, x), \quad n=0,1,2,
$$

as $k \rightarrow \infty$. Then, on the set $\{v>0\}$, the function

$$
f^{*}:(t, x) \mapsto B\left(t, x, v^{t, x}\right)+q^{*}\left(t, x, v^{t, x}\right)
$$

satisfies

$$
\limsup _{k \rightarrow \infty} f^{*}\left(t_{k}, x_{k}\right) \leqslant f^{*}(t, x)
$$

Similarly, the function

$$
f_{*}:(t, x) \mapsto B\left(t, x, v^{t, x}\right)+q_{*}\left(t, x, v^{t, x}\right)
$$

satisfies

$$
\liminf _{k \rightarrow \infty} f_{*}\left(t_{k}, x_{k}\right) \leqslant f_{*}(t, x) .
$$

In particular, if $v^{t, x} \in C_{1}\left(\mathcal{O}_{T}\right),(t, x) \in \mathcal{O}_{T}$ is a continuum of functions, then $f^{*}$ is lower semicontinuous and $f_{*}$ is upper semicontinuous in $\mathcal{O}_{T}$.

(iii) Suppose $v \in C_{1}\left(\mathcal{O}_{T}\right)$ is $C^{1,2}$ at $(t, x)$, and satisfies the subsolution [supersolution] inequality in the classical sense at $(t, x)$. Then $v$ also satisfies the subsolution [supersolution] inequality in the viscosity sense at $(t, x)$.

(iv) Conversely, suppose $v$ is a subsolution [supersolution] in the viscosity sense, and $\hat{v} \geqslant v$ $[\hat{v} \leqslant v]$ is $C^{1,2}$ at $(t, x)$. Then $\hat{v}$ satisfies the subsolution [supersolution] inequality in the classical sense at $(t, x)$.

(v) If 1.1 has a classical solution $v \in C^{1,2}\left(\mathcal{O}_{T}\right)$, then it is also a viscosity solution.

(vi) Suppose $v \in U S C\left(\mathcal{O}_{T}\right)\left[v \in L S C\left(\mathcal{O}_{T}\right)\right]$ satisfies the supersolution [subsolution] property for $x>0$. Then $v$ satisfies the supersolution [subsolution] property at $x=0$.

Proof. To confirm (i), first observe that because $v_{1}(t, x)=v_{2}(t, x)$ we have either (I) $q\left(t, x, v_{1}\right)=$ $q\left(t, x, v_{1}\right)=0$ or (II) $q\left(t, x, v_{1}\right)=c\left(t, x, v_{1}\right)$ and $q\left(t, x, v_{2}\right)=c\left(t, x, v_{1}\right)$. In case (I) the claim holds by monotonicity of the integral term $B$. For (II), from the assumptions we have $v_{1}(t, x)=$ $v_{2}(t, x), \partial_{x} v_{1}(t, x)=\partial_{x} v_{2}(t, x)$, which implies also that

$$
B\left(t, x, v_{2}\right)-B\left(t, x, v_{1}\right)=\int_{\mathbb{R}}\left[v_{2}\left(t, x e^{z}\right)-v_{1}\left(t, x e^{z}\right)\right] v_{t}(\mathrm{~d} z),
$$

and in particular the integral on the right hand side is well defined. Now, an elementary estimation shows that for $f^{+}(x):=\max \{0, f(x)\}$ we have $f^{+}(x)-g^{+}(x) \leqslant(f(x)-g(x))^{+}$for any functions $f, g$. Using this and $H_{*}\left(v\left(t, x e^{z}\right)-\left(K-x e^{z}\right)\right)\left(v\left(t, x e^{z}\right)-\left(K-x e^{z}\right)\right)=\left(v\left(t, x e^{z}\right)-\left(K-x e^{z}\right)\right)^{+}$, we deduce 


$$
\begin{aligned}
B(t, & \left.x, v_{2}\right)+q\left(t, x, v_{2}\right)-B\left(t, x, v_{1}\right)-q\left(t, x, v_{1}\right) \\
& =B\left(t, x, v_{2}\right)-B\left(t, x, v_{1}\right)+\left(r K-d x-D\left(t, x, v_{2}\right)\right)^{+}-\left(r K-d x-D\left(t, x, v_{1}\right)\right)^{+} \\
& \leqslant B\left(t, x, v_{2}\right)-B\left(t, x, v_{1}\right)+\left(D\left(t, x, v_{1}\right)-D\left(t, x, v_{2}\right)\right)^{+} \\
& \leqslant \int_{\mathbb{R}}\left[v_{2}\left(t, x e^{z}\right)-v_{1}\left(t, x e^{z}\right)\right] v_{t}(\mathrm{~d} z)+\int_{\mathbb{R}}\left(v_{1}\left(t, x e^{z}\right)-v_{2}\left(t, x e^{z}\right)\right)^{+} v_{t}(\mathrm{~d} z)=0,
\end{aligned}
$$

where the last equality follows from $v_{1} \geqslant v_{2}$. To verify 6.6, note that on the one hand, monotonicity of $B+q$ implies

$$
B\left(t, x, v_{1}+C\right)+q\left(t, x, v_{1}+C\right) \geqslant B\left(t, x, v_{1}\right)+q\left(t, x, v_{1}\right)
$$

On the other hand,

$$
B\left(t, x, v_{1}+C\right)+q\left(t, x, v_{1}+C\right)=B\left(t, x, v_{1}\right)+q\left(t, x, v_{1}+C\right) \leqslant B\left(t, x, v_{1}\right)+q\left(t, x, v_{1}\right) .
$$

Next, (ii) follows by continuity of $v$, the assumptions on the family $v^{t, x}$ and the continuity properties of the integral terms. Claim (iii) follows by standard application of the necessary criteria for maxima of differentiable functions and monotonicity properties of the operator. Claim (v) is a direct consequence of (iii). To prove (iv) for the case of a subsolution, we pick $\bar{\phi} \in C^{1,2}\left(\mathcal{O}_{T}\right)$ such that $\bar{\phi} \geqslant v, v(t, x)=\bar{\phi}(t, x), \partial_{t} v(t, x)=\partial_{t} \bar{\phi}(t, x), \partial_{x} v(t, x)=\partial_{x} \bar{\phi}(t, x)$, and $\partial_{x}^{2} v(t, x)=$ $\partial_{x}^{2} \bar{\phi}(t, x)$. This can be done by the construction of Evans (see [73, Proposition 4.5.4]). Moreover, let $\left\{v_{k}\right\}_{k=1}^{\infty} \subset C_{1}^{\infty}\left(\mathcal{O}_{T}\right)$ be such that $v_{k} \downarrow v$ almost everywhere as $k \rightarrow \infty$. Let $\mathcal{X}_{k}$ be a smooth function such that $0 \leqslant \mathcal{X}_{k} \leqslant 1, \mathcal{X}_{k}=1$ in a ball with radius $1 / 2 k$ and center at $(t, x)$, and $\mathcal{X}_{k}=0$ outside a ball with radius $1 / k$ and center at $(t, x)$. Then

$$
\phi_{k}(s, y):=\mathcal{X}_{k}(y) \bar{\phi}(s, y)+\left(1-\mathcal{X}_{k}(y)\right) v_{k}(s, y)
$$

defines a sequence of test functions such that $\partial_{x}^{n} \phi_{k}(t, x)=\partial_{x}^{n} v(t, x), n=0,1,2$, and $\phi^{k} \downarrow v$ everywhere as $k \rightarrow \infty$. Note especially that by monotone convergence

$$
\lim _{k \rightarrow \infty} B\left(t, x, \phi_{k}\right)=B(t, x, v),
$$

so the sequence of integrals has a well defined limit. The claim then follows from (ii), and proof for the case of a supersolution is symmetric. Claim (vi) holds by adapting arguments in Lemma 4.1 of [20, p. 288] and using the semicontinuity of $f^{*}, f_{*}$.

\section{Existence}

For the existence result, the following lemma will be useful.

LEMMA 6 The pay-off function $g$ is a viscosity subsolution of the semilinear Black and Scholes equation 1.1.

Proof. We prove the lemma for the put option $g(x)=(K-x)^{+}$, the proof for the call option is similar. We will show that, in fact, $g$ satisfies the equation in the classical sense whenever $x \neq K$. Furthermore, if $x=K$ there is no smooth function $\phi \in C^{1,2}\left(\mathcal{O}_{T}\right)$ such that $\phi \geqslant g$ and $\phi(t, K)=$ $g(K)$. Then the claim follows by Proposition 5 . 
Let $x \neq K$ and note that $q^{*}(t, x, g)=c(t, x, g)$ and $\bar{D}(t, x, g)=0$ everywhere. We have four cases to consider. If $x<K$ and $r K-d x \geqslant 0$ (Case I), we have

$$
\mathcal{L}_{B S} g(x)+B(t, x, g)+q^{*}(t, x, g)=0 .
$$

If $x>K$ and $r K-d x \leqslant 0$ (Case II), then $g=0$ in a neighborhood of $x$, so the claim holds trivially. If $x<K$ and $r K-d x \leqslant 0$ (Case III), then

$$
\mathcal{L}_{B S} g(x)+B(t, x, g)+q^{*}(t, x, g)=-(r K-d x) \geqslant 0 .
$$

If $x>K$ and $r K-d x \geqslant 0$ (Case IV), then

$$
\mathcal{L}_{B S} g(x)+B(t, x, g)+q^{*}(t, x, g)=\int_{\mathbb{R}} g\left(x e^{z}\right) v_{t}(\mathrm{~d} z)+r K-d x \geqslant 0 .
$$

The following lemma states that the linear part of equation (5.7) comes from the characteristic operator of the space-time process $(u, S(u))$, and is a simple consequence of the Itô formula for semimartingales and the Dynkin formula.

Lemma 7 For $n \in \mathbb{N}$, let $\theta_{n}$ be the exit time for the space-time process $(u, S(u)), u \in[0, T]$, from a ball with radius $1 / n$ and center at $(t, x)$. Then, for $\phi \in C_{1}^{1,2}\left(\overline{\mathcal{O}_{T}}\right)$,

$$
\frac{\mathbb{E}^{t, x}\left[e^{-r\left(\theta_{n}-t\right)} \phi\left(\theta_{n}, S\left(\theta_{n}\right)\right)\right]-\phi(t, x)}{\mathbb{E}^{t, x}\left[\theta_{n}\right]-t} \stackrel{n \rightarrow \infty}{\longrightarrow} \mathcal{L}_{B S} \phi(t, x)+B(t, x, \phi) .
$$

THEOREM 8 The value function $v(t, x)$ defined in (4.1) is a viscosity solution of the terminal value problem 5.7)-(5.8).

Proof. Continuity of the value function follows from Proposition 2 and it is clear from the definition that the value function satisfies the terminal condition. It remains to prove that $v$ is a subsolution and a supersolution of the semilinear equation (5.7).

We begin with the supersolution property. Let $\phi \in C_{1}^{1,2}\left(\mathcal{O}_{T}\right)$ and $(t, x) \in \mathcal{O}_{T}$ be such that $v \geqslant \phi$ and $v(t, x)=\phi(t, x)$. For $n \in \mathbb{N}$, let $\theta_{n}$ be the exit time of the space-time process $(u, S(u))$ from a ball with radius $1 / n$ and center at $(t, x)$. Using $v \geqslant \phi, v(t, x)=g(x)$, 4.47, and Lemma7. we deduce

$$
\begin{aligned}
0 & =\frac{v(t, x)-\phi(t, x)}{\mathbb{E}^{t, x}\left[\theta_{n}\right]-t} \geqslant \frac{\mathbb{E}^{t, x}\left[e^{-r\left(\theta_{n}-t\right)} v\left(\theta_{n}, S\left(\theta_{n}\right)\right)\right]-\phi(t, x)}{\mathbb{E}^{t, x}\left[\theta_{n}\right]-t} \\
& \geqslant \frac{\mathbb{E}^{t, x}\left[e^{-r\left(\theta_{n}-t\right)} \phi\left(\theta_{n}, S\left(\theta_{n}\right)\right)\right]-\phi(t, x)}{\mathbb{E}^{t, x}\left[\theta_{n}\right]-t} \rightarrow \mathcal{L}_{B S} \phi(t, x)+B(t, x, \phi)
\end{aligned}
$$

as $n \rightarrow \infty$. Notice also that $\phi(t, x)=v(t, x) \geqslant g(x)$, so $q_{*}(t, x, \phi)=0$ in $[0, T) \times \mathbb{R}_{+}$. Thus,

$$
\mathcal{L}_{B S} \phi(t, x)+B(t, x, \phi)+q_{*}(t, x, \phi) \leqslant 0,
$$

so $v$ is a supersolution of the semilinear Black and Scholes equation (5.7).

Let us next prove that $v$ is a subsolution. Let $\phi \in C_{1}^{1,2}\left(\overline{\mathcal{O}_{T}}\right)$ and $(t, x) \in \mathcal{O}_{T}$ be such that $v \leqslant \phi$ and $v(t, x)=\phi(t, x)$. Assume first that $(t, x)$ is in the continuation region, i.e., $v(t, x)>g(x)$. Then $q^{*}(t, x, \phi)=0$, and 4.5 implies

$$
\mathcal{L}_{B S} \phi(t, x)+B(t, x, \phi) \geqslant 0 .
$$


Assume finally that $(t, x)$ is in the stopping region (so that $\phi(t, x)=v(t, x)=g(x)$ ). Since $\phi(t, x)=v(t, x)=g(x)$ and $\phi \geqslant v \geqslant g$, we conclude by the subsolution property of $g$ (Lemma 6) that

$$
\mathcal{L}_{B S} \phi(t, x)+B(t, x, \phi)+q^{*}(t, x, \phi) \geqslant 0,
$$

which yields the subsolution property of $v$, concluding our proof.

REMARK 9 Notice that the above proof applies to both the call and the put option, once it is recognized that the corresponding payoff $g$ is a subsolution of the given equation in both cases. The introduction of the localizing stopping time $\theta_{n}$ is necessary for call options, while for put options one could work out a simpler proof using compactly supported test functions and the infinitesimal generator of $\left(u, S^{t, x}(u)\right)$.

\section{A strong comparison principle}

In this section, we follow a quite self-contained approach outlined in [73] for proving comparison principles. To adapt this approach to our partial integro-differential equation, we also borrow ideas from [9, 20, 45, 62, 67, 68].

As mentioned in the introduction, Jakobsen and Karlsen discuss in [45] some issues concerning the applicability of Ishii's lemma in connection with integro-differential equations. We note that their results are not applicable as such here because of the discontinuity in the reaction term. The subsequent work of Arisawa [4], [5], and the recent paper by Barles and Imbert [10] also apply maximum principles. Rather than reworking through the rather long proofs of these types of abstract maximum principles, we work with the semiconvex approximations that are the main tools behind such results (see [33]) and allow for a rather direct proof. We mention that insight gained in this way is used in [71] to show how Ishii's lemma can in fact be applied for integro-differential equations if this is done in a careful manner.

We will construct approximations of the sub- and supersolutions which are then sub- and supersolutions of an approximate semilinear Black and Scholes equation, following the standard approach of using sup- and inf convolutions [7, 37, 73]. Let $v \in U S C\left(\overline{\mathcal{O}_{T}}\right)$ satisfy $v(t, x) \leqslant$ $L(1+x)$ in $\overline{\mathcal{O}_{T}}$ and let $\gamma<1 / 2 \sqrt{L}$. We denote the sup convolution of $v$ by $v^{\gamma}$. Similarly, the inf convolution is denoted by $v_{\gamma}$, which is meaningful as long as $v \in \operatorname{LSC}\left(\overline{\mathcal{O}_{T}}\right)$ satisfies $v(t, x) \geqslant-L(1+x)$ in $\overline{\mathcal{O}_{T}}$ and $\gamma<1 / 2 \sqrt{L}$. We refer to [7, 37, 73] for precise definitions. These approximations have some well-known properties that will be useful later, which we list in the following lemma [7, 37, 73].

LEMma 10 (i) Let $v \in U S C\left(\overline{\mathcal{O}_{T}}\right)$ satisfy $0 \leqslant v(t, x) \leqslant L(1+x)$ in $\overline{\mathcal{O}_{T}}$ and let $0<\gamma<1 / 2 L^{1 / 2}$. Then $0 \leqslant v^{\gamma}(t, x) \leqslant 2 L(1+x)$ and $v^{\gamma}(t, x)+\left(1 / 2 \gamma^{2}\right)\left(t^{2}+x^{2}\right)$ is convex (i.e., $v^{\gamma}$ is semiconvex). Define $C(t, x):=(4 L(1+x)-2 v(t, x))^{1 / 2}$. If $(t, x) \in \mathcal{O}_{T}$ is such that $\operatorname{dist}\left((t, x), \partial \mathcal{O}_{T}\right)>$ $\sqrt{2} \gamma C(t, x)$, then there exists $(\hat{t}, \hat{x})$ such that $|(t, x)-(\hat{t}, \hat{x})| \leqslant \sqrt{2} \gamma C(t, x)$ and

$$
v^{\gamma}(t, x)=v(\hat{t}, \hat{x})-\frac{1}{2 \gamma^{2}}\left[|t-\hat{t}|^{2}+|x-\hat{x}|^{2}\right] .
$$

(ii) Let $v \in L S C\left(\overline{\mathcal{O}_{T}}\right)$ satisfy $0 \leqslant v(t, x) \leqslant C(1+x)$ in $\overline{\mathcal{O}_{T}}$ and let $0<\gamma<1 / 2 L^{1 / 2}$. Then $0 \leqslant v_{\gamma}(t, x) \leqslant 2 L(1+x)$ and $v_{\gamma}(t, x)-\left(1 / 2 \gamma^{2}\right)\left(t^{2}+x^{2}\right)$ is concave (i.e., $v_{\gamma}$ is semiconcave). If $(t, x) \in \mathcal{O}_{T}$ is such that $\operatorname{dist}\left((t, x), \partial \mathcal{O}_{T}\right)>\sqrt{2} \gamma C(t, x)$, then there exists $(\hat{t}, \hat{x})$ such that 
$|(t, x)-(\hat{t}, \hat{x})| \leqslant \sqrt{2} \gamma C(t, x)$ and

$$
v_{\gamma}(t, x)=v(\hat{t}, \hat{x})+\frac{1}{2 \gamma^{2}}\left[|t-\hat{t}|^{2}+|x-\hat{x}|^{2}\right] .
$$

(iii) Finally, $v^{\gamma} \downarrow v$ and $v_{\gamma} \uparrow v$ pointwise as $\gamma \rightarrow 0$.

In particular, from Alexandrov's theorem it follows that the sup and inf convolutions are $C^{1,2}$ almost everywhere. We refer to [7, 37, 73] for this fact and proofs of results like those in Lemma 10.

Let $G$ denote the Hamiltonian

$$
G_{(*)}^{*}(t, x, q, p, P, v)=r x p+\frac{1}{2} \sigma^{2}(t) x^{2} P-r q+B(t, x, v)+q_{(*)}^{*}(t, x, v) .
$$

Now, if $v$ is a function satisfying the assumptions of Lemma 10 , we define, for fixed $\gamma$,

$$
\mathcal{O}_{T}^{v, \gamma}:=\left\{(t, x) \in \mathcal{O}_{T} \mid \operatorname{dist}\left((t, x), \partial \mathcal{O}_{T}\right)>\sqrt{2} \gamma C(t, x)\right\},
$$

where $C(t, x)$ is defined in Lemma 10 . Moreover, let $\tau_{k}^{l}$ denote the shift operator defined by

$$
\tau_{k}^{l} \phi(t, x):=\phi(t+k, x+l)
$$

for any function $\phi$ and any $(t, x),(t+h, x+h)$ in the domain of definition of $\phi$. To introduce suitable approximations of the semilinear Black and Scholes equation we will need the operators

$$
\begin{aligned}
G^{\gamma}(t, x, q, p, P, v)=\sup _{(s, y)}\left\{r y p+\frac{1}{2} \sigma^{2}(s) y^{2} P-r q+B\left(s, y, \tau_{t-s}^{x-y} v\right)\right. \\
\left.\quad+q^{*}\left(s, y, \tau_{t-s}^{x-y} v\right)||(t, x)-(s, y) \mid \leqslant \sqrt{2} \gamma C(t, x)\right\}
\end{aligned}
$$

and

$$
\begin{aligned}
G_{\gamma}(t, x, q, p, P, v)=\inf _{(s, y)}\left\{r y p+\frac{1}{2} \sigma^{2}(s) y^{2} P-r q+B\left(s, y, \tau_{t-s}^{x-y} v\right)\right. \\
\left.\quad+q_{*}\left(s, y, \tau_{t-s}^{x-y} v\right)||(t, x)-(s, y) \mid \leqslant \sqrt{2} \gamma C(t, x)\right\} .
\end{aligned}
$$

Notice that both $G^{\gamma}$ and $G_{\gamma}$ inherit the same monotonicity in $q, P$ as the original, unperturbed operator. Moreover, the property shown in Proposition 5(i) is preserved for the nonlocal part of the operators.

The following lemma shows that the semiconvex approximations of sub- and supersolutions satisfy an equation modified by the above operators.

LEMMA 11 (a) Suppose $v \in U S C\left(\overline{\mathcal{O}_{T}}\right), 0 \leqslant v(t, x) \leqslant L(1+x)$, is a subsolution of the semilinear equation 5.7 and $v^{\gamma}$ is the sup convolution of $v$ for $0<\gamma<1 / 2 L^{1 / 2}$. If $v^{\gamma}$ is $C^{1,2}$ at $(t, x)$, then

$$
\partial_{t} v^{\gamma}(t, x)+G^{\gamma}\left(t, x, v^{\gamma}(t, x), \partial_{x} v^{\gamma}(t, x), \partial_{x}^{2} v^{\gamma}(t, x), v^{\gamma}\right) \geqslant 0 \quad \text { in } \mathcal{O}_{T}^{v, \gamma} \cap\left\{v^{\gamma}>0\right\} .
$$

(b) Suppose $v \in L S C\left(\overline{\mathcal{O}_{T}}\right), 0 \leqslant v \leqslant L(1+x)$, is a viscosity supersolution of the semilinear equation 5.7) and $v_{\gamma}$ is the inf convolution of $v$ for $0<\gamma<1 / 2 L^{1 / 2}$. If $v_{\gamma}$ is $C^{1,2}$ at $(t, x)$, then

$$
\partial_{t} v_{\gamma}(t, x)+G_{\gamma}\left(t, x, v_{\gamma}(t, x), \partial_{x} v_{\gamma}(t, x), \partial_{x}^{2} v_{\gamma}(t, x), v_{\gamma}\right) \leqslant 0 \quad \text { in } \mathcal{O}_{T}^{v, \gamma} \text {. }
$$


Proof. We give the proof for (a); the proof of (b) is similar. Suppose $v^{\gamma}$ is $C^{1,2}$ at $(\bar{t}, \bar{x}) \in \mathcal{O}_{T}^{v, \gamma}$. Suppose that $(\hat{t}, \hat{x})$ satisfies 8.1$)$. For any $(t, x)$, we have

$$
\begin{aligned}
v(s, y)-\frac{1}{2 \gamma^{2}}\left[|t-s|^{2}+|x-y|^{2}\right]-v(t, x) & \leqslant 0=v^{\gamma}(\bar{t}, \bar{x})-v^{\gamma}(\bar{t}, \bar{x}) \\
& =v(\hat{t}, \hat{x})-\frac{1}{2 \gamma^{2}}\left[|\bar{t}-\hat{t}|^{2}+|\bar{x}-\hat{x}|^{2}\right]-v^{\gamma}(\bar{t}, \bar{x}) .
\end{aligned}
$$

Choosing $(t, x)=(s-\hat{t}+\bar{t}, y-\hat{x}+\bar{x})$ in this inequality implies that the mapping

$$
v(s, y)-v^{\gamma}(s-\hat{t}+\bar{t}, y-\hat{x}+\bar{x})
$$

attains a global maximum at $(s, y)=(\hat{t}, \hat{x})$. Let us define a function

$$
\hat{v}(s, y):=v^{\gamma}(s-\hat{t}+\bar{t}, y-\hat{x}+\bar{x})+v(\hat{t}, \hat{x})-v^{\gamma}(\bar{t}, \bar{x}) .
$$

Since $(\bar{t}, \bar{x}) \in \mathcal{O}_{T}^{v, \gamma}$ and $|(\bar{t}, \bar{x})-(\hat{t}, \hat{x})| \leqslant \sqrt{2} \gamma C(\bar{t}, \bar{x})$, the function is well defined for all $y>0$ and $s$ in a neighborhood of $\hat{t}$. By the above estimates, $\hat{v} \geqslant v$ with $\hat{v}(\hat{t}, \hat{x})=v(\hat{t}, \hat{x})$. Furthermore, $\hat{v}$ is differentiable at $(\hat{t}, \hat{x})$ with $\partial_{t} \hat{v}(\hat{t}, \hat{x})=\partial_{t} v^{\gamma}(\bar{t}, \bar{x}), \partial_{x} \hat{v}(\hat{t}, \hat{x})=\partial_{x} v^{\gamma}(\bar{t}, \bar{x})$ and $\partial_{x}^{2} \hat{v}(\hat{t}, \hat{x})=$ $\partial_{x}^{2} v^{\gamma}(\bar{t}, \bar{x})$. Thus, by Proposition 5(iii), $\hat{v}$ satisfies the subsolution inequality in the classical sense so that

$$
\begin{aligned}
& \partial_{t} v^{\gamma}(\bar{t}, \bar{x})+(r-d) \hat{x} \partial_{x} v^{\gamma}(\bar{t}, \bar{x})+\frac{1}{2} \sigma^{2}(\hat{t}) \hat{x}^{2} \partial_{x}^{2} v^{\gamma}(\bar{t}, \bar{x})-r v^{\gamma}(\bar{t}, \bar{x})+B(\hat{t}, \hat{x}, \hat{v})+q^{*}(\hat{t}, \hat{x}, \hat{v}) \\
& =\partial_{t} \hat{v}(\hat{t}, \hat{x})+(r-d) \hat{x} \partial_{x} \hat{v}(\hat{t}, \hat{x})+\frac{1}{2} \sigma^{2}(\hat{t}) \hat{x}^{2} \partial_{x}^{2} \hat{v}(\hat{t}, \hat{x})-r \hat{v}(\hat{t}, \hat{x})+B(\hat{t}, \hat{x}, \hat{v})+q^{*}(\hat{t}, \hat{x}, \hat{v}) \geqslant 0
\end{aligned}
$$

and by definition of $G^{\gamma}$, we obtain

$$
\partial_{t} v^{\gamma}(\bar{t}, \bar{x})+G^{\gamma}\left(\bar{t}, \bar{x}, v^{\gamma}(\bar{t}, \bar{x}), \partial_{x} v^{\gamma}(\bar{t}, \bar{x}), \partial_{x}^{2} v^{\gamma}(\bar{t}, \bar{x}), v^{\gamma}\right) \geqslant 0,
$$

where we have used 6.6 with $C=v(\hat{t}, \hat{x})-v^{\gamma}(\bar{t}, \bar{x}) \geqslant 0$.

Our main result is a comparison principle for the terminal value problem (5.7)- 5.8). The comparison principle is strong in the sense that it applies for a class of semicontinuous functions satisfying a natural growth condition. Besides implying uniqueness of the viscosity solution, the comparison principle is useful in proving convergence of approximate solutions to the equation. Here we apply the approximation procedures given in this section and which are at the heart of the more abstract maximum principles. The proof depends fundamentally on the monotonicity property of the whole nonlocal part

$$
v \mapsto B(t, x, v)+q(t, x, v)
$$

of the operator.

For a function $v$ which is $C_{1}^{1,2}$ at $(t, x)$, define

$$
\bar{D}(t, x, v):=\int_{\mathbb{R}} H^{*}(g(x)-v(t, x)) H_{*}\left(v\left(t, x e^{z}\right)-g\left(x e^{z}\right)\right)\left(v\left(t, x e^{y}\right)-\left(K-x e^{z}\right)\right) v_{t}(\mathrm{~d} z)
$$

and

$$
\underline{D}(t, x, v):=\int_{\mathbb{R}} H_{*}(g(x)-v(t, x)) H_{*}\left(v\left(t, x e^{z}\right)-g\left(x e^{z}\right)\right)\left(v\left(t, x e^{y}\right)-\left(K-x e^{z}\right)\right) v_{t}(\mathrm{~d} z) .
$$


These definitions will be useful for book-keeping purposes in our proof. In this regard, we note especially that, the corresponding source terms satisfy

$$
q^{*}(t, x, v)=H^{*}(g(x)-v(t, x))(r K-d x-\bar{D}(t, x, v))^{+}
$$

and

$$
q_{*}(t, x, v)=H_{*}(g(x)-v(t, x))(r K-d x-\underline{D}(t, x, v))^{+} .
$$

THEOREM 12 (Comparison principle) Suppose that $\underline{v} \in U S C_{1}^{+}\left(\overline{\mathcal{O}_{T}}\right)$ is a subsolution and $\bar{v} \in$ $\operatorname{LSC}_{1}^{+}\left(\overline{\mathcal{O}_{T}}\right)$ is a supersolution of the semilinear BS equation, satisfying

$$
\underline{v}(T, x) \leqslant \bar{v}(T, x), \quad x \in[0, \infty) .
$$

Then

$$
\underline{v} \leqslant \bar{v} \quad \text { on }[0, T] \times \mathbb{R}_{+} .
$$

Proof. Let $\mu>0$ and define $\bar{v}^{\mu}(t, x):=\bar{v}(t, x)+\mu(T-t)$. We prove the claim holds for $\bar{v}^{\mu}$, and the main claim follows by taking $\mu \rightarrow 0$.

As noted before, we first assume the claim does not hold. Thus, suppose there exist $\delta>0$ and $(\bar{t}, \bar{x}) \in[0, T] \times \mathbb{R}_{+}$such that

$$
\underline{v}(\bar{t}, \bar{x}) \geqslant \bar{v}^{\mu}(\bar{t}, \bar{x})+3 \delta .
$$

Let $\underline{v}^{\gamma}, \bar{v}_{\gamma}$ denote the sup and inf convolutions of $\underline{v}$ and $\bar{v}$, respectively. Furthermore, let $\bar{v}_{\gamma}^{\mu}(t, x):=$ $\bar{v}_{\gamma}(\bar{t}, x)+\mu(T-t)$. Then, since $\underline{v}^{\gamma} \downarrow \underline{v}$ and $\bar{v}_{\gamma}^{\mu} \bar{v}^{\mu}$ as $\gamma \downarrow 0$, for $\gamma>0$ the functions $\underline{v}^{\gamma}$ and $\bar{v}_{\gamma}^{\mu}$ satisfy

$$
\underline{v}^{\gamma}(\bar{t}, \bar{x})-\bar{v}_{\gamma}^{\mu}(\bar{t}, \bar{x}) \geqslant \underline{v}(\bar{t}, \bar{x})-\bar{v}^{\mu}(\bar{t}, \bar{x}) \geqslant 3 \delta,
$$

so

$$
\underline{v}^{\gamma}(\bar{t}, \bar{x}) \geqslant \bar{v}_{\gamma}^{\mu}(\bar{t}, \bar{x})+3 \delta
$$

also. We define

$$
\Phi(t, x, y):=\underline{v}(t, x)-\bar{v}^{\mu}(t, y)-\psi(t, x, y), \quad(t, x, y) \in[0, T] \times \mathbb{R}_{+} \times \mathbb{R}_{+},
$$

and

$$
\Phi_{\gamma}(t, x, y):=\underline{v}^{\gamma}(t, x)-\bar{v}_{\gamma}^{\mu}(t, y)-\psi(t, x, y), \quad(t, x, y) \in[0, T] \times \mathbb{R}_{+} \times \mathbb{R}_{+},
$$

for any $\gamma>0$ where

$$
\psi(t, x, y)=\frac{\alpha}{2}(x-y)^{2}+\frac{\epsilon}{2} e^{\lambda(T-t)}\left(x^{2}+y^{2}\right) .
$$

We note also that $\Phi_{0}(t, x, y):=\lim _{\gamma \rightarrow 0} \Phi_{\gamma}(t, x, y)=\Phi$. It is standard in viscosity solutions theory [34, Lemma 3.1, p. 15] to see that (for each fixed $\gamma, \epsilon$ ) there exists a sequence of maxima $\left(t_{\alpha}, x_{\alpha}, y_{\alpha}\right)$ of $\Phi_{\gamma}$ that converge to a limit point $\left(t_{\epsilon}, x_{\epsilon}, y_{\epsilon}\right)$. Furthermore, the maxima $\left(t_{\alpha}, x_{\alpha}, y_{\alpha}\right)$ satisfy

$$
x_{\alpha}-y_{\alpha} \rightarrow 0 \text { and } \alpha\left|x_{\alpha}-y_{\alpha}\right|^{2} \rightarrow 0
$$

as $\alpha \rightarrow \infty$. Note that we have dropped the dependence on $\epsilon$ and $\gamma$ for notational convenience. 
Observe that

$$
\Phi_{\gamma}\left(t_{\alpha}, x_{\alpha}, y_{\alpha}\right) \geqslant \Phi_{\gamma}(\bar{t}, \bar{x}, \bar{x})=\underline{v}^{\gamma}(\bar{t}, \bar{x})-\bar{v}_{\gamma}^{\mu}(\bar{t}, \bar{x})-\epsilon e^{\lambda(T-\bar{t})} \bar{x}^{2} \geqslant 2 \delta>0
$$

for any $\alpha>1, \gamma \geqslant 0$, and any $\epsilon>0$ that is small enough. This implies

$$
\underline{v}^{\gamma}\left(t_{\alpha}, x_{\alpha}\right) \geqslant \bar{v}_{\gamma}^{\mu}\left(t_{\alpha}, y_{\alpha}\right)+2 \delta>0
$$

for any $\alpha>1, \gamma \geqslant 0$ and any $\epsilon$ sufficiently small.

Let us now look at the special case $t_{\epsilon}=T$. Note that

$$
\underline{v}(\bar{t}, \bar{x})-\bar{v}^{\mu}(\bar{t}, \bar{x})-\epsilon e^{\lambda(T-\bar{t})} \bar{x}^{2} \leqslant \Phi\left(t_{\alpha}, x_{\alpha}, y_{\alpha}\right) \leqslant \underline{v}\left(t_{\alpha}, x_{\alpha}\right)-\bar{v}^{\mu}\left(t_{\alpha}, y_{\alpha}\right) .
$$

By the upper semicontinuity of $\underline{v},-\bar{v}^{\mu}$, and since $\left.\underline{v}\right|_{t=T} \leqslant \bar{v}_{t=T}^{\mu}$ on $[0, \infty)$, we can send $\alpha \uparrow \infty$ in this inequality to obtain $\underline{v}(\bar{t}, \bar{x})-\bar{v}^{\mu}(\bar{t}, \bar{x})-\epsilon e^{\lambda(T-\bar{t})} \bar{x}^{2} \leqslant 0$, which contradicts $(8.11$. Hence we may assume from now on that $t_{\epsilon}<T$. Then $t_{\alpha}<T$ for any $\alpha$ sufficiently large.

Let $Q^{\epsilon}$ be a compact and convex set in $\overline{\mathcal{O}_{T}}$ such that the subsequence of maximum points $\left(t_{\alpha}, x_{\alpha}, y_{\alpha}\right)$ is contained in $Q^{\epsilon}$ for $\alpha>1,0<\gamma<1 / 2$. Then the restriction of $\psi$ to $Q^{\epsilon}$ is smooth with bounded derivatives, which implies its semiconcavity. Thus $\Phi$ is semiconvex on $Q$. Consequently, for small $\gamma>0$,

$$
\hat{\Phi}_{\gamma}(t, x, y):=\Phi_{\gamma}(t, x, y)-s\left(\left|t-t_{\alpha}\right|^{2}+\left|x-x_{\alpha}\right|^{2}+\left|y-y_{\alpha}\right|^{2}\right)
$$

is semiconvex on $Q^{\epsilon}$ and attains a strict maximum at $\left(t_{\alpha}, x_{\alpha}, y_{\alpha}\right)$. By the lemmas of Alexandrov and Jensen (see p. 202 in [73]), there exist $q, p, \hat{p} \in \mathbb{R}$ (depending on $\gamma>0$ ) with

$$
|q|+|p|+|\hat{p}| \leqslant \gamma
$$

and $\left(\hat{t}_{\alpha}, \hat{x}_{\alpha}, \hat{y}_{\alpha}\right)$ with

$$
\left|\hat{t}_{\alpha}-t_{\alpha}\right|+\left|\hat{x}_{\alpha}-x_{\alpha}\right|+\left|\hat{y}_{\alpha}-y_{\alpha}\right| \leqslant \gamma
$$

such that

$$
\hat{\Phi}_{\gamma}(t, x, y)+q t+p x+\hat{p} y
$$

attains a maximum at $\left(\hat{t}_{\alpha}, \hat{x}_{\alpha}, \hat{y}_{\alpha}\right)$, at which $\underline{v}^{\gamma}(t, x)-\bar{v}_{\gamma}^{\mu}(t, y)$ is twice differentiable. By the firstand second-order necessary conditions for a maximum point we must have

$$
\begin{aligned}
\partial_{t} \underline{v}^{\gamma}\left(\hat{t}_{\alpha}, \hat{x}_{\alpha}\right)-\partial_{t} \bar{v}_{\gamma}^{\mu}\left(\hat{t}_{\alpha}, \hat{y}_{\alpha}\right) & =-\lambda \frac{\epsilon}{2} e^{\lambda\left(T-\hat{t}_{\alpha}\right)}\left(\hat{x}_{\alpha}^{2}+\hat{y}_{\alpha}^{2}\right)+2 \gamma\left(\hat{t}_{\alpha}-t_{\alpha}\right)-q, \\
\partial_{x} \underline{v}^{\gamma}\left(\hat{t}_{\alpha}, \hat{x}_{\alpha}\right) & =\alpha\left(\hat{x}_{\alpha}-\hat{y}_{\alpha}\right)+\epsilon e^{\lambda\left(T-\hat{t}_{\alpha}\right)} \hat{x}_{\alpha}+2 \gamma\left(\hat{x}_{\alpha}-x_{\alpha}\right)-p, \\
\partial_{y} \bar{v}_{\gamma}^{\mu}\left(\hat{t}_{\alpha}, \hat{y}_{\alpha}\right) & =-\alpha\left(\hat{x}_{\alpha}-\hat{y}_{\alpha}\right)-\epsilon e^{\lambda\left(T-\hat{t}_{\alpha}\right)} \hat{y}_{\alpha}-2 \gamma\left(\hat{y}_{\alpha}-y_{\alpha}\right)+\hat{p},
\end{aligned}
$$

and

$$
\left(\begin{array}{cc}
\partial_{x}^{2} \underline{v}^{\gamma}\left(\hat{t}_{\alpha}, \hat{x}_{\alpha}\right) & 0 \\
0 & -\partial_{y}^{2} \bar{v}_{\gamma}^{\mu}\left(\hat{t}_{\alpha}, \hat{y}_{\alpha}\right)
\end{array}\right) \leqslant \alpha\left(\begin{array}{cc}
1 & -1 \\
-1 & 1
\end{array}\right)+\left(\epsilon e^{\lambda\left(T-\hat{t}_{\alpha}\right)}+2 \gamma\right) I,
$$

where $I$ is the $2 \times 2$ identity matrix. Also, by Lemma 11

$$
\partial_{t} \underline{v}^{\gamma}\left(\hat{t}_{\alpha}, \hat{x}_{\alpha}\right)+G^{\gamma}\left(\hat{t}_{\alpha}, \hat{x}_{\alpha}, \underline{v}^{\gamma}\left(\hat{t}_{\alpha}, \hat{x}_{\alpha}\right), \partial_{x} \underline{v}^{\gamma}\left(\hat{t}_{\alpha}, \hat{x}_{\alpha}\right), \partial_{x}^{2} \underline{v}^{\gamma}\left(\hat{t}_{\alpha}, \hat{x}_{\alpha}\right), \underline{v}^{\gamma}\right) \geqslant 0
$$


and, using monotonicity of $G_{\gamma}$, it is straightforward to notice that $\bar{v}_{\gamma}^{\mu}$ satisfies

$$
\partial_{t} \bar{v}_{\gamma}^{\mu}\left(\hat{t}_{\alpha}, \hat{y}_{\alpha}\right)+G_{\gamma}\left(\hat{t}_{\alpha}, \hat{y}_{\alpha}, \bar{v}_{\gamma}^{\mu}\left(\hat{t}_{\alpha}, \hat{y}_{\alpha}\right), \partial_{x} \bar{v}_{\gamma}^{\mu}\left(\hat{t}_{\alpha}, \hat{y}_{\alpha}\right), \partial_{x}^{2} \bar{v}_{\gamma}^{\mu}\left(\hat{t}_{\alpha}, \hat{y}_{\alpha}\right), \bar{v}_{\gamma}^{\mu}\right) \leqslant-\mu .
$$

By the definition of $G^{\gamma}$ and $G_{\gamma}$, we can find a point $\left(\bar{t}_{\alpha}, \bar{x}_{\alpha}, \bar{y}_{\alpha}\right)$ with

$$
\left|\bar{t}_{\alpha}-\hat{t}_{\alpha}\right|+\left|\bar{x}_{\alpha}-\hat{x}_{\alpha}\right|+\left|\bar{y}_{\alpha}-\hat{y}_{\alpha}\right| \leqslant K_{\epsilon} \gamma^{2}
$$

for some constant $K_{\epsilon}$ only depending on $\epsilon$, such that

$$
\begin{aligned}
\mu \leqslant & \partial_{t} \underline{v}^{\gamma}\left(\hat{t}_{\alpha}, \hat{x}_{\alpha}\right)+G^{\gamma}\left(\hat{t}_{\alpha}, \hat{x}_{\alpha}, \underline{v}^{\gamma}\left(\hat{t}_{\alpha}, \hat{x}_{\alpha}\right), \partial_{x} \underline{v}^{\gamma}\left(\hat{t}_{\alpha}, \hat{x}_{\alpha}\right), \partial_{x}^{2} \underline{v}^{\gamma}\left(\hat{t}_{\alpha}, \hat{x}_{\alpha}\right), \underline{v}^{\gamma}\right) \\
& -\partial_{t} \bar{v}_{\gamma}^{\mu}\left(\hat{t}_{\alpha}, \hat{y}_{\alpha}\right)-G_{\gamma}\left(\hat{t}_{\alpha}, \hat{y}_{\alpha}, \bar{v}_{\gamma}^{\mu}\left(\hat{t}_{\alpha}, \hat{y}_{\alpha}\right), \partial_{y} \bar{v}_{\gamma}^{\mu}\left(\hat{t}_{\alpha}, \hat{y}_{\alpha}\right), \partial_{y}^{2} \bar{v}_{\gamma}^{\mu}\left(\hat{t}_{\alpha}, \hat{y}_{\alpha}\right), \bar{v}_{\gamma}^{\mu}\right) \\
= & \partial_{t} \underline{v}^{\gamma}\left(\hat{t}_{\alpha}, \hat{x}_{\alpha}\right)+G^{*}\left(\bar{t}_{\alpha}, \bar{x}_{\alpha}, \underline{v}^{\gamma}\left(\hat{t}_{\alpha}, \hat{x}_{\alpha}\right), \partial_{x} \underline{v}^{\gamma}\left(\hat{t}_{\alpha}, \hat{x}_{\alpha}\right), \partial_{x}^{2} \underline{v}^{\gamma}\left(\hat{t}_{\alpha}, \hat{x}_{\alpha}\right), \tau_{\hat{t}_{\alpha}-\bar{t}}^{\hat{x}_{\alpha}-\bar{v}} \underline{v}^{\gamma}\right) \\
& -\partial_{t} \bar{v}_{\gamma}^{\mu}\left(\hat{t}_{\alpha}, \hat{y}_{\alpha}\right)-G_{*}\left(\bar{t}_{\alpha}, \bar{y}_{\alpha}, \bar{v}_{\gamma}^{\mu}\left(\hat{t}_{\alpha}, \hat{y}_{\alpha}\right), \partial_{y} \bar{v}_{\gamma}^{\mu}\left(\hat{t}_{\alpha}, \hat{y}_{\alpha}\right), \partial_{y}^{2} \bar{v}_{\gamma}^{\mu}\left(\hat{t}_{\alpha}, \hat{y}_{\alpha}\right), \tau_{\hat{y}_{\alpha}-\bar{t}}^{\hat{y}_{\alpha}-\bar{v}} \bar{v}_{\gamma}^{\mu}\right) \\
= & I_{1}+I_{2}+I_{3}+I_{4}+I_{5}+I_{6},
\end{aligned}
$$

where

$$
\begin{aligned}
& I_{1}=\partial_{t} \underline{v}^{\gamma}\left(\hat{t}_{\alpha}, \hat{x}_{\alpha}\right)-\partial_{t} \bar{v}_{\gamma}^{\mu}\left(\hat{t}_{\alpha}, \hat{y}_{\alpha}\right), \\
& I_{2}=(r-d)\left[\bar{x}_{\alpha} \partial_{x} \underline{v}^{\gamma}\left(\hat{t}_{\alpha}, \hat{x}_{\alpha}\right)-\bar{y}_{\alpha} \partial_{y} \bar{v}_{\gamma}^{\mu}\left(\hat{t}_{\alpha}, \hat{y}_{\alpha}\right)\right], \\
& I_{3}=\frac{1}{2} \sigma^{2}\left(\bar{t}_{\alpha}\right)\left[\bar{x}_{\alpha}^{2} \partial_{x}^{2} \underline{v}^{\gamma}\left(\hat{t}_{\alpha}, \hat{x}_{\alpha}\right)-\bar{y}_{\alpha}^{2} \partial_{y}^{2} \bar{v}_{\gamma}^{\mu}\left(\hat{t}_{\alpha}, \hat{y}_{\alpha}\right)\right], \\
& I_{4}=r\left[\bar{v}_{\gamma}^{\mu}\left(\hat{t}_{\alpha}, \hat{y}_{\alpha}\right)-\underline{v}^{\gamma}\left(\hat{t}_{\alpha}, \hat{x}_{\alpha}\right)\right], \\
& I_{5}=B\left(\bar{t}_{\alpha}, \bar{x}_{\alpha}, \tau_{\hat{t}_{\alpha}-\bar{x}_{\alpha}}^{\hat{x}_{\alpha}-\bar{v}^{\gamma}} \underline{v}^{\gamma}\right)-B\left(\bar{t}_{\alpha}, \bar{y}_{\alpha}, \tau_{\hat{t}_{\alpha}-\hat{y}_{\alpha}-\bar{y}_{\alpha}}^{\hat{v}_{\alpha}} \bar{v}_{\gamma}^{\mu}\right), \\
& I_{6}=q^{*}\left(\bar{t}_{\alpha}, \bar{x}_{\alpha}, \tau_{\hat{t}_{\alpha}-\bar{t}_{\alpha}}^{\hat{x}_{\alpha}} \underline{v}^{\gamma}\right)-q_{*}\left(\bar{t}_{\alpha}, \bar{y}_{\alpha}, \tau_{\hat{t}_{\alpha}-\bar{y}_{\alpha}}^{\hat{y}_{\alpha}} \bar{v}_{\gamma}^{\mu}\right) .
\end{aligned}
$$

We now make estimates, first for the local terms $I_{1}-I_{4}$ and then for the nonlocal ones $I_{5}, I_{6}$. For the local part, the only nontrivial term is the second order term $I_{3}$. For this, notice that fully utilizing the information given by the Jacobian leads to the estimate

$$
\begin{aligned}
& I_{3}=\frac{1}{2} \sigma^{2}\left(\bar{t}_{\alpha}\right)\left\{\left(\begin{array}{cc}
\bar{x}_{\alpha} & \left.\bar{y}_{\alpha}\right)
\end{array}\right)\left(\begin{array}{cc}
\partial_{x}^{2} \underline{v}^{\gamma}\left(\hat{t}_{\alpha}, \hat{x}_{\alpha}\right) & 0 \\
0 & -\partial_{y}^{2} \bar{v}_{\gamma}^{\mu}\left(\hat{t}_{\alpha}, \hat{y}_{\alpha}\right)
\end{array}\right)\left(\begin{array}{l}
\bar{x}_{\alpha} \\
\bar{y}_{\alpha}
\end{array}\right)\right\} \\
& \leqslant \frac{1}{2} \sigma^{2}\left(\bar{t}_{\alpha}\right)\left\{\left(\begin{array}{ll}
\bar{x}_{\alpha} & \bar{y}_{\alpha}
\end{array}\right)\left(\alpha\left(\begin{array}{cc}
1 & -1 \\
-1 & 1
\end{array}\right)+\epsilon e^{\lambda\left(T-\hat{t}_{\alpha}\right)} I_{2}\right)\left(\begin{array}{l}
\bar{x}_{\alpha} \\
\bar{y}_{\alpha}
\end{array}\right)+2 \gamma\left(\bar{x}_{\alpha}^{2}-\bar{y}_{\alpha}^{2}\right)\right\} \\
& =\frac{1}{2} \sigma^{2}\left(\bar{t}_{\alpha}\right)\left\{\alpha\left(\bar{x}_{\alpha}-\bar{y}_{\alpha}\right)^{2}+\epsilon e^{\lambda\left(T-\hat{t}_{\alpha}\right)}\left(\bar{x}_{\alpha}^{2}+\bar{y}_{\alpha}^{2}\right)+2 \gamma\left(\bar{x}_{\alpha}^{2}-\bar{y}_{\alpha}^{2}\right)\right\} .
\end{aligned}
$$

Combining this with some straightforward calculations and also the observation $\lim _{\gamma \rightarrow 0} I_{4} \leqslant-r \delta$, we get the estimate

$$
\limsup _{\gamma \downarrow 0, \alpha \uparrow \infty}\left(I_{1}+I_{2}+I_{3}+I_{4}\right) \leqslant \epsilon\left(r+\sigma^{2}\left(t_{\epsilon}\right)-d-\lambda\right) e^{\lambda\left(T-t_{\epsilon}\right)} x_{\epsilon}^{2} \leqslant 0
$$

by choosing $\lambda$ large enough. 
It remains to estimate the nonlocal part $I_{5}+I_{6}$. First note that $\tau_{\hat{t}_{\alpha}-\hat{t}_{\alpha}}^{\hat{\hat{x}}_{\alpha}} \underline{\bar{v}}^{\gamma}\left(\bar{t}_{\alpha}, \bar{x}_{\alpha}\right)=\underline{v}^{\gamma}\left(\hat{t}_{\alpha}, \hat{x}_{\alpha}\right)$ and $\tau_{\hat{t}_{\alpha}-\bar{t}_{\alpha}}^{\hat{y}_{\alpha}-\bar{y}_{\alpha}} \bar{v}_{\gamma}^{\mu}\left(\bar{t}_{\alpha}, \bar{y}_{\alpha}\right)=\bar{v}_{\gamma}^{\mu}\left(\hat{t}_{\alpha}, \hat{y}_{\alpha}\right)$. For $I_{5}$, the integrand equals

$$
\begin{aligned}
f_{5}= & \tau_{\hat{x}_{\alpha}-\bar{t}_{\alpha}}^{\hat{x}_{\alpha}} \underline{v}^{\gamma}\left(\bar{t}_{\alpha}, \bar{x}_{\alpha} e^{z}\right)-\tau_{\hat{y}_{\alpha}-\bar{y}_{\alpha}}^{\hat{y}_{\alpha}} \bar{y}_{\gamma} \bar{v}_{\gamma}^{\mu}\left(\bar{t}_{\alpha}, \bar{y}_{\alpha} e^{z}\right)-\left(\underline{v}^{\gamma}\left(\hat{t}_{\alpha}, \hat{x}_{\alpha}\right)-\bar{v}_{\gamma}^{\mu}\left(\hat{t}_{\alpha}, \hat{y}_{\alpha}\right)\right) \\
& -\left[\alpha\left(\hat{x}_{\alpha}-\hat{y}_{\alpha}\right)\left(\bar{x}_{\alpha}-\bar{y}_{\alpha}\right)+\epsilon e^{\lambda\left(T-\hat{t}_{\alpha}\right)}\left(\bar{x}_{\alpha} \hat{x}_{\alpha}+\bar{y}_{\alpha} \hat{y}_{\alpha}\right)\right]\left(e^{z}-1\right) \\
& +\left[2 \gamma \bar{x}_{\alpha}\left(\hat{x}_{\alpha}-x_{\alpha}\right)-p \bar{x}_{\alpha}+2 \gamma \bar{y}_{\alpha}\left(\hat{y}_{\alpha}-y_{\alpha}\right)-\hat{p} \bar{y}_{\alpha}\right]\left(e^{z}-1\right) .
\end{aligned}
$$

To get a nicer expression than the above, we first take the limit $\gamma \downarrow 0$, make some estimates for the limit and then apply Fatou's lemma:

$$
\begin{aligned}
\limsup _{\gamma \downarrow 0} f_{5} \leqslant & \underline{v}\left(t_{\alpha}, x_{\alpha} e^{z}\right)-\bar{v}^{\mu}\left(t_{\alpha}, y_{\alpha} e^{z}\right)-\left(\underline{v}\left(t_{\alpha}, x_{\alpha}\right)-\bar{v}\left(t_{\alpha}, y_{\alpha}\right)\right) \\
& -\left[\alpha\left(x_{\alpha}-y_{\alpha}\right)^{2}+\epsilon e^{\lambda\left(T-t_{\alpha}\right)}\left(x_{\alpha}^{2}+y_{\alpha}^{2}\right)^{2}\right]\left(e^{z}-1\right) \\
= & \Phi\left(t_{\alpha}, x_{\alpha} e^{z}, y_{\alpha} e^{z}\right)-\Phi\left(t_{\alpha}, x_{\alpha}, y_{\alpha}\right)+\psi\left(t_{\alpha}, x_{\alpha}, y_{\alpha}\right)\left(e^{z}-1\right)^{2} \\
\leqslant & \psi\left(t_{\alpha}, x_{\alpha}, y_{\alpha}\right)\left(e^{z}-1\right)^{2},
\end{aligned}
$$

where the last inequality follows by the maximality of $\left(t_{\alpha}, x_{\alpha}, y_{\alpha}\right)$. By Fatou's lemma we then have

$$
\limsup _{\gamma \downarrow 0} I_{5} \leqslant \psi\left(t_{\alpha}, x_{\alpha}, y_{\alpha}\right) \int_{\mathbb{R}}\left(e^{z}-1\right)^{2} v_{t_{\alpha}}(\mathrm{d} z) .
$$

For the nonstandard term $I_{6}$, we begin by choosing $\gamma$ small and $\alpha$ large so that $\left|\bar{x}_{\alpha}-\bar{y}_{\alpha}\right| \leqslant \delta / 2$. Note also that by continuity and 8.12 , we get

$$
\underline{v}^{\gamma}\left(\hat{t}_{\alpha}, \hat{x}_{\alpha}\right) \geqslant \bar{v}_{\gamma}^{\mu}\left(\hat{t}_{\alpha}, \hat{y}_{\alpha}\right)+\delta>0
$$

for small $\gamma$ and large $\alpha$. Thus

$$
\begin{aligned}
g\left(\bar{y}_{\alpha}\right)-\bar{v}_{\gamma}^{\mu}\left(\hat{t}_{\alpha}, \hat{y}_{\alpha}\right) & =g\left(\bar{x}_{\alpha}\right)-\bar{v}^{\mu}\left(\hat{t}_{\alpha}, \hat{y}_{\alpha}\right)+\left(g\left(\bar{y}_{\alpha}\right)-g\left(\bar{x}_{\alpha}\right)\right) \\
& \geqslant g\left(\bar{x}_{\alpha}\right)-\bar{v}_{\gamma}^{\mu}\left(\hat{t}_{\alpha}, \hat{y}_{\alpha}\right)-\delta / 2 \geqslant g\left(\bar{x}_{\alpha}\right)-\underline{v}^{\gamma}\left(\hat{t}_{\alpha}, \hat{x}_{\alpha}\right)+\delta / 2 .
\end{aligned}
$$

By comparing possible values of $H^{*}$ and $H_{*}$ one then derives the estimates

$$
\begin{aligned}
& I_{6} \leqslant \max \left\{0, H^{*}\left(g\left(\bar{x}_{\alpha}\right)-\underline{v}^{\gamma}\left(\hat{t}_{\alpha}, \hat{x}_{\alpha}\right)\right)\left[\left(r K-d \bar{x}_{\alpha}-\bar{D}\left(\bar{t}_{\alpha}, \bar{x}_{\alpha}, \tau_{\hat{t}_{\alpha}-\bar{t}_{\alpha}}^{\hat{x}_{\alpha}-\bar{x}_{\alpha}} \underline{v}^{\gamma}\right)\right)^{+}\right.\right. \\
&\left.\left.-\left(r K-d \bar{y}_{\alpha}-\underline{D}\left(\bar{t}_{\alpha}, \bar{y}_{\alpha}, \tau_{\hat{t}_{\alpha}-\bar{y}_{\alpha}}^{\hat{y}_{\alpha}-\bar{y}_{\alpha}} \bar{v}_{\gamma}^{\mu}\right)\right)^{+}\right]\right\} \\
& \leqslant d\left(\bar{y}_{\alpha}-\bar{x}_{\alpha}\right)^{+}+\max \left\{0, H^{*}\left(g\left(\bar{x}_{\alpha}\right)-\underline{v}^{\gamma}\left(\hat{t}_{\alpha}, \hat{x}_{\alpha}\right)\right)\left[\underline{D}\left(\bar{t}_{\alpha}, \bar{y}_{\alpha}, \tau_{\hat{y}_{\alpha}-\bar{t}_{\alpha}} \overline{\hat{y}}_{\alpha} \bar{v}_{\gamma}^{\mu}\right)-\bar{D}\left(\bar{t}_{\alpha}, \bar{x}_{\alpha}, \tau_{\hat{x}_{\alpha}-\bar{t}_{\alpha}} \underline{\hat{x}}^{\hat{x}_{\alpha}} \underline{v}^{\gamma}\right)\right]\right\} .
\end{aligned}
$$

The first term vanishes when we take $\gamma \rightarrow 0, \alpha \rightarrow \infty$. By comparing possible values of the integrands we see that the second term is less than or equal to

$$
\int_{|z|>\hat{\kappa}} \max \left\{0,-\left(\tau_{\hat{t}_{\alpha}-\bar{t}_{\alpha}}^{\hat{x}_{\alpha}-\bar{x}_{\alpha}} \underline{v}^{\gamma}\left(\bar{t}_{\alpha}, \bar{x}_{\alpha} e^{z}\right)-\tau_{\hat{t}_{\alpha}-\bar{t}_{\alpha}}^{\hat{x}_{\alpha}-\bar{y}_{\alpha}} \bar{v}_{\gamma}^{\mu}\left(\bar{t}_{\alpha}, \bar{y}_{\alpha} e^{z}\right)\right)+\left(\bar{x}_{\alpha}-\bar{y}_{\alpha}\right) e^{z}\right\} v_{\bar{t}_{\alpha}}(\mathrm{d} z)
$$

for some fixed $\hat{\kappa}>0$. Defining

$$
f_{6}:=\max \left\{0,1_{|z|>\hat{\kappa}}\left[-\left(\tau_{\hat{t}_{\alpha}-\bar{x}_{\alpha}}^{\hat{x}_{\alpha}-\bar{x}_{\alpha}} \underline{v}^{\gamma}\left(\bar{t}_{\alpha}, \bar{x}_{\alpha} e^{z}\right)-\tau_{\hat{t}_{\alpha}-\hat{t}_{\alpha}}^{\hat{x}_{\alpha}-\bar{y}_{\alpha}} \bar{v}_{\gamma}^{\mu}\left(\bar{t}_{\alpha}, \bar{y}_{\alpha} e^{z}\right)\right)+\left(\bar{x}_{\alpha}-\bar{y}_{\alpha}\right) e^{z}\right]\right\}
$$


and recalling calculations for $f_{5}$, we may estimate

$$
\begin{aligned}
\limsup _{\gamma \downarrow 0}\left(f_{5}+f_{6}\right) \leqslant & \Phi\left(t_{\alpha}, x_{\alpha} e^{z}, y_{\alpha} e^{z}\right)-\Phi\left(t_{\alpha}, x_{\alpha}, y_{\alpha}\right)+\psi\left(t_{\alpha}, x_{\alpha}, y_{\alpha}\right)\left(e^{z}-1\right)^{2} \\
& +\max \left\{0,-1_{|z|>\hat{\kappa}}\left[\Phi\left(t_{\alpha}, x_{\alpha} e^{z}, y_{\alpha} e^{z}\right)+\left(x_{\alpha}-y_{\alpha}\right) e^{z}\right]\right\} \\
\leqslant & \max \left\{0, \psi\left(t_{\alpha}, x_{\alpha}, y_{\alpha}\right)\left(e^{z}-1\right)^{2}+1_{|z|>\hat{\kappa}}\left(x_{\alpha}-y_{\alpha}\right) e^{z}\right\},
\end{aligned}
$$

where we have once again used maximality and nonnegativity of $\Phi\left(t_{\alpha}, x_{\alpha}, y_{\alpha}\right)$. Thus

$$
\begin{aligned}
\limsup _{\gamma \downarrow 0}\left(I_{5}+I_{6}\right) \leqslant & d\left(y_{\alpha}-x_{\alpha}\right)^{+}+\psi\left(t_{\alpha}, x_{\alpha}, y_{\alpha}\right) \int_{\mathbb{R}}\left(e^{z}-1\right)^{2} v_{t_{\alpha}}(\mathrm{d} z) \\
& +\max \left\{0,\left(x_{\alpha}-y_{\alpha}\right) \int_{|z|>\hat{\kappa}} e^{z} v_{t_{\alpha}}(\mathrm{d} z)\right\} .
\end{aligned}
$$

Thus

$$
\lim \sup \left(I_{5}+I_{6}\right) \leqslant \epsilon e^{\lambda\left(T-t_{\epsilon}\right)}\left(\int_{\mathbb{R}}\left(e^{z}-1\right)^{2} v_{t_{\epsilon}}(\mathrm{d} z)\right) x_{\epsilon}^{2}
$$

as we take $\gamma \rightarrow 0$ and $\alpha \rightarrow \infty$, in this order. Combining 8.16 with 8.18 , we obtain

$$
\lim \sup \sum_{i=1}^{6} I_{i} \leqslant \epsilon e^{\lambda\left(T-t_{\epsilon}\right)}\left(r-d+\sigma^{2}\left(t_{\epsilon}\right)+K_{\epsilon}-\lambda\right) x_{\epsilon}^{2},
$$

where

$$
K_{\epsilon}=\int_{\mathbb{R}}\left(e^{z}-1\right)^{2} v_{t_{\epsilon}}(\mathrm{d} z)
$$

By choosing $\lambda>0$ large we finally obtain the contradiction

$$
0<\mu \leqslant 0 .
$$

Note carefully that we did not need to take $\epsilon \rightarrow 0$, but only to choose $\epsilon$ small. The contradiction is made possible by the use of the free parameter $\lambda$, which again is actually available to us by the parabolicity of the equation.

Since the viscosity solution $v$ is both a sub- and supersolution, the comparison principle implies uniqueness for the semilinear Black and Scholes equation (1.1). We furthermore see that $v$ is the smallest supersolution satisfying $v \geqslant g$, which is in accordance with the classical characterization of value functions of optimal stopping problems. It is interesting to note that we do not need to assume $v \geqslant g$ a priori, but that this information is embedded in the operator and follows from the comparison principle. Finally, the square integrability assumption on the price process was made so that we could arrive at the inequality 8.17 in the above proof. This could possibly be handled by a modification of the penalizations $\psi_{\epsilon, \gamma}$ that only grows sublinearly outside of a compact but sufficiently large set.

\section{Acknowledgements}

We thank Fred Espen Benth for suggesting the topic and his efforts in carefully reading and making valuable comments on this paper. 
This work was supported in part by the Research Council of Norway through the project "Integro-PDEs: Numerical methods, Analysis, and Applications to Finance" and an Outstanding Young Investigators Award of K. H. Karlsen. The authors thank an anonymous referee for many valuable comments leading to improvements of the paper.

\section{REFERENCES}

1. Alili, L., \& Kyprianou, A. Some remarks on first passage of Lévy processes, the American put and pasting principles. Ann. Appl. Probab. 15 (2005), 2062-2080. Zbl 1083.60034 MR 2152253

2. AMADORI, A. L. Obstacle problem for nonlinear integro-differential operators arising in option pricing. Quaderno IAC Q21-000 (2000).

3. Applebaum, D. Lévy Processes and Stochastic Calculus. Cambridge Univ. Press, Cambridge (2004). Zbl 1073.60002 MR 2072890

4. ARISAWA, M. A new definition of viscosity solutions for a class of second-order degenerate elliptic integro-differential equations. Ann. Inst. H. Poincaré Anal. Non Linéaire 23 (2006), 695-711. Zbl 1105.45004 MR 2259613

5. ARISAWA, M. Corrigendum in the comparison theorems in "A new definition of viscosity solutions for a class of second-order degenerate elliptic integro-differential equations". Ann. Inst. H. Poincaré Anal. Non Linéaire 24 (2006), 167-169. Zbl 1125.45008 MR 2286563

6. Ash, R. B. Real Analysis and Probability. Academic Press, New York (1972). MR 0435320

7. Bardi, M., \& CAPUzzo-Dolcetta, I. Optimal Control and Viscosity Solutions of Hamilton-JacobiBellman Equations. Birkhäuser, Boston (1997). Zbl 0890.49011 MR 1484411

8. BARLES, G. Convergence of numerical schemes for degenerate parabolic equations arising in finance theory. In: Numerical Methods in Finance, Cambridge Univ. Press (1997), 1-21. Zbl 0898.90015 MR 1470506

9. BARles, G., BuCKDAhn, R., \& PARdouX, E. Backward stochastic differential equations and integralpartial differential equations. Stoch. Stoch. Rep. 60 (1997), 57-83. Zbl 0878.60036 MR 1436432

10. Barles, G., \& Imbert, C. Second-order elliptic integro-differential equations: viscosity solutions theory revisited. Ann. Inst. H. Poincaré Anal. Non Linéaire 25 (2008), 567-585. Zbl 1155.45004 MR 2422079

11. Barles, G., \& Souganidis, P. E. Convergence of approximation schemes for nonlinear second order equations. Asymptotic Anal. 4 (1991), 271-283. Zbl 0729.65077 MR 1115933

12. BARNDORFF-NiELSEN, O. E. Exponentially decreasing distributions for the logarithm of particle size. Proc. Roy. Soc. London 353 (1977), 401-419.

13. Barndorff-Nielsen, O. E. Processes of normal inverse Gaussian type. Finance Stoch. 2 (1998), 41-68. Zbl 0894.90011 MR 1804664

14. Barone-Adesi, G. The saga of the American put. J. Banking Finance 29 (2005), 2909-2918.

15. BAYRAKTAR, E. A proof of the smoothness of the finite time horizon American put option for jump diffusions. SIAM J. Control Optim. 48 (2009), 551-572. MR 2486083

16. Bayraktar, E., \& XING, H. Analysis of the optimal exercise boundary of American options for jump diffusions. SIAM J. Math. Anal. 41 (2009), 825-860. MR 2515786

17. BAYRAKTAR, E., \& XING, H. Regularity of the optimal stopping problem for Lévy processes with nondegenerate diffusions. Submitted (2009).

18. Bensoussan, A., \& Lions, J.-L. Applications of Variational Inequalities in Stochastic Control. NorthHolland (1982). Zbl 0478.49002 MR 0653144

19. Bensoussan, A., \& LiONS, J.-L. Impulse Control and Quasi-Variational Inequalities. Gauthier-Villars (1984). MR 0756234 
20. Benth, F. E., Karlsen, K. H., \& Reikvam, K. A semilinear Black and Scholes partial differential equation for valuing American options. Finance Stoch. 7 (2003), 277-298. Zbl 1064.60080 MR 1994910

21. Benth, F. E., KARlsen, K. H., \& Reikvam, K. A semilinear Black and Scholes partial differential equation for valuing American options: approximate solutions and convergence. Interfaces Free Bound. 6 (2004), 379-404. Zbl 1068.35190 MR 2111562

22. Bertoin, J. Lévy Processes. Cambridge Univ. Press, Cambridge (1996). Zbl 0861.60003 MR 1406564

23. Blumenthal, R. M., \& Getoor, R.K. Markov Processes and Potential Theory. Academic Press, New York and London (1968). Zbl 0169.49204 MR 0264757

24. Brennan, M. J., \& Schwartz, E. S. The valuation of American put options. J. Finance 32 (1977), 449-462.

25. Caffarelli, L. A., Salsa, S., \& Silvestre, L. Regularity estimates for the solution and the free boundary of the obstacle problem for the fractional Laplacian. Invent. Math. 171 (2008), 425-461. Zbl 1148.35097 MR 2367025

26. CARR, P., \& Hirsa, A. Why be backward? Forward equations for American options. Risk, January 2003, 103-107. MR 2343216

27. Carr, P., Jarrow, R., \& Myneni, R. Alternative characterizations of American put options. J. Math. Finance 2 (1992), 87-106.

28. Chiarella, C., \& Ziogas, A. Pricing American option on jump-diffusion processes using Fourier Hermite series expansions. UTS Quantitative Finance Research Centre research paper, 145 (2005).

29. Chiarella, C., \& Ziogas, A. Pricing American option under stochastic volatility. UTS Quantitative Finance Research Centre research paper (2005).

30. Chiarella, C., \& Ziogas, A. A Fourier transform analysis of the American call option on assets driven by jump-diffusion processes. UTS Quantitative Finance Research Centre research paper, 174 (2006).

31. Chiarella, C., Ziogas, A., \& Kucera, A. A survey of integral representation of American option prices. UTS Quantitative Finance Research Centre research paper, 118 (2004).

32. Cont, R., \& TANKov, P. Financial Modelling with Jump Processes. Chapman \& Hall, London (2004). Zbl 1052.91043 MR 2042661

33. Crandall, M. G., \& Ishit, H. The maximum principle for semicontinuous functions. Differential Integral Equations 3 (1990), 1001-1014. Zbl 0723.35015 MR 1073054

34. Crandall, M. G., IshiI, H., \& LiOns, P.-L. User's guide to viscosity solutions of second order partial differential equations. Bull. Amer. Math. Soc. 27 (1992), 1-67. Zbl 0755.35015 MR 1118699

35. Eberlein, E. Application of generalized hyperbolic Lévy motions to finance. In: O. E. BarndorffNielsen et al. (eds.), Lévy Processes: Theory and Applications, Birkhäuser (2001), 319-337. Zbl 0982.60045 MR 1833703

36. Eberlein, E., \& Papapantoleon, A. Symmetries and pricing of exotic options in Lévy models. In: A. Kyprianou et al. (eds.), Exotic Option Pricing and Advanced Lévy Models, Wiley (2005), 99-128. MR 2343210

37. Fleming, W. H., \& Soner, H. M. Controlled Markov Processes and Viscosity Solutions. Springer, New York (2006). Zbl 1105.60005 MR 2179357

38. Forsyth, P. A., \& VetZal, K. R. Quadratic convergence for valuing American options using a penalty method. SIAM J. Comput. Sci. 23 (2002), 2095-2122. Zbl 1020.91017 MR 1923727

39. Gikhman, I. I., \& Skorohod, A. V. The Theory of Stochastic Processes II. Springer (2004). Zbl 1068.60005 MR 2058260

40. Hirsa, A., \& Madan, D. B. Pricing American options under variance gamma. J. Comput. Finance 7 (2003/04), 63-80. 
41. IsHII, H. Hamilton-Jacobi equations with discontinuous Hamiltonians on arbitrary open sets. Bull. Fac. Sci. Engrg. Chuo Univ. 28 (1985), 33-77. Zbl 0937.35505 MR 0845397

42. IsHII, H. On uniqueness and existence of viscosity solutions of fully nonlinear second-order elliptic PDEs. Comm. Pure Appl. Math. 42 (1989), 15-45. Zbl 0645.35025 MR 0973743

43. ISHII, H., \& LIONS, P.-L. Viscosity solutions of fully nonlinear second-order elliptic partial differential equations. J. Differential Equations 83 (1990), 26-78. Zbl 0708.35031 MR 1031377

44. JACOD, J., \& Shiryaev, A. N. Limit Theorems for Stochastic Processes. 2nd ed., Springer (2003). Zbl 1018.60002 MR 1943877

45. JAKOBSEN, E., \& KARLSEN, K. H. A maximum principle applicable to partial integro-differential equations. Nonlinear Differential Equations Appl. 13 (2006), 137-165. Zbl 1105.45006 MR 2243708

46. Jamshidian, F. An analysis of American options. Rev. Futures Markets 11 (1992), 72-80.

47. Jensen, R. The maximum principle for viscosity solutions of fully nonlinear second order partial differential equations. Arch. Ration. Mech. Anal. 101 (1988), 1-27. Zbl 0708.35019 MR 0920674

48. Karatzas, I., \& Shreve, S. E. Methods of Mathematical Finance. Springer (1998). Zbl 0941.91032 MR 1640352

49. Kholodnyi, V. A nonlinear partial integro-differential equation for the American option in the whole domain of the state variable. Nonlinear Anal. 30 (1997), 5059-5070. Zbl 0911.90029 MR 1726008

50. Kluge, W. Time-inhomogeneous Lévy processes in interest rate and credit risk models. $\mathrm{PhD}$ thesis, Univ. of Freiburg (2005); http://www.freidok.uni-freiburg.de/volltexte/2090/pdf/diss.pdf Zbl 1135.60017

51. Kou, S. A jump-diffusion model for option pricing. Management Sci. 48 (2002), 1086-1101.

52. Lamberton, D., \& Mikou, M. The critical price for the American put in an exponential Lévy model. Finance Stoch. 12 (2008), 561-581. Zbl pre05591787 MR 2447412

53. Madan, D. The variance gamma process and option pricing. Eur. Finance Rev. 2 (1998), 79-105. Zbl 0937.91052

54. Mandelbrot, B. The variation of certain speculative prices. J. Business 36 (1963), 394-419.

55. MCKeAn, H. P. Appendix: a free boundary problem for the heat equation arising from a problem in mathematical economics. Indust. Management Rev. 6 (1965), 32-39.

56. Merton, R. Option pricing when underlying stock returns are discontinuous. J. Financial Economics 3 (1976), 125-144. Zbl 1131.91344

57. Myneni, R. The pricing of the American option. Ann. Appl. Probab. 2 (1992), 1-23. Zbl 0753.60040 MR 1143390

58. Nielsen, B. F., Skavhaug, O., \& Tveito, A. Penalty and front fixing methods for the numerical solution of American option problems. J. Comput. Finance $\mathbf{5}$.

59. Lapyere, B., Jaillet, P., \& LAmberton, D. Variational inequalities and the pricing of American options. Acta Appl. Math. 21 (1990), 263-289. Zbl 0714.90004 MR 1096582

60. Peskir, G., \& ShiRyaev, A. Optimal Stopping and Free-Boundary Problems. Birkhäuser (2006). Zbl 1115.60001 MR 2256030

61. Pham, H. Optimal stopping, free boundary, and American option in a jump diffusion model. Appl. Math. Optim. 35, 145-164. Zbl 0866.60038 MR 1424787

62. PHAM, H. Optimal stopping of controlled jump diffusion processes: A viscosity solutions approach. J. Math. Systems Estim. Control 8 (1998), 1-27. Zbl $0899.60039 \mid$ MR 1650147

63. RAIBLE, S. Lévy processes in finance: theory, numerics and empirical facts. PhD thesis, Univ. of Freiburg (2000); http://deposit.ddb.de/cgi-bin/dokserv?idn=961285192 Zbl 0966.60044

64. Revuz, D., \& Yor, M. Continuous Martingales and Brownian Motion. 3rd ed., Springer, Berlin (1999). Zbl 0917.60006 MR 1725357

65. Samuelson, P. A. Rational theory of warrant pricing. Indust. Management Rev. 6 (1965), 13-31. 
66. Sato, K. Lévy Processes and Infinitely Divisible Distributions. Cambridge Univ. Press, Cambridge (1999). Zbl 0973.60001 MR 1739520

67. SAYAh, A. Équations d'Hamilton-Jacobi du premier ordre avec termes intégro-différentiels. I. Unicité des solutions de viscosité. Comm. Partial Differential Equations 16 (1991), 1057-1074. Zbl 0742.45004 MR 1116853

68. SAYAH, A. Équations d'Hamilton-Jacobi du premier ordre avec termes intégro-différentiels. II. Existence de solutions de viscosité. Comm. Partial Differential Equations 16 (1991), 1075-1093. Zbl 0742.45005 MR 1116854

69. ShiRyaev, A. N. Optimal Stopping Rules. Springer, Berlin (1978). Zbl 0391.60002 MR 2374974

70. SONER, H. M. Optimal control with state-space constraint. II. SIAM J. Control Optim. 24 (1986), 11101122. Zbl 0619.49013 MR 0861089

71. WALlin, O. Viscosity solutions theory for tolling agreements. Preprint, Univ. of Oslo Pure Math. E-Print 4 (2007); http://www.math.uio.no/eprint/pure_math/2008/04-08.html

72. YANG, C. R., JiAng, L. S. \& BIAN, B. J. Free boundary and American options in a jump-diffusion model. Eur. J. Appl. Math. 17 (2006), 95-127. Zbl 1160.91364 MR 2228973

73. Yong, J., \& ZHou, X.Y. Stochastic Controls: Hamiltonian Systems and HJB Equations. Springer, Berlin (1999). Zbl 0943.93002 MR 1696772

74. ZHU, S.-P. An exact and explicit solution for the valuation of American put options. Quant. Finance 6 (2006), 229-242. Zbl 1136.91468 MR 2241106

75. Zvan, R., Forsyth, P. A., \& VetZal, K. R. Penalty methods for American options with stochastic volatility. J. Comput. Appl. Math. 91 (1998), 199-218. Zbl 0945.65005 MR 1628686 